\title{
Ventral Midline Thalamus Is Critical for Hippocampal-Prefrontal Synchrony and Spatial Working Memory
}

\author{
Henry L. Hallock, ${ }^{1}$ Arick Wang, ${ }^{2}$ and Amy L. Griffin ${ }^{1}$ \\ ${ }^{1}$ Department of Psychological and Brain Sciences, University of Delaware, Newark, Delaware 19716, and 2Department of Psychology, Emory University, \\ Atlanta, Georgia 30322
}

Maintaining behaviorally relevant information in spatial working memory (SWM) requires functional synchrony between the dorsal hippocampus and medial prefrontal cortex (mPFC). However, the mechanism that regulates synchrony between these structures remains unknown. Here, we used a unique dual-task approach to compare hippocampal-prefrontal synchrony while rats switched between an SWM-dependent task and an SWM-independent task within a single behavioral session. We show that task-specific representations in MPFC neuronal populations are accompanied by SWM-specific oscillatory synchrony and directionality between the dorsal hippocampus and mPFC. We then demonstrate that transient inactivation of the reuniens and rhomboid $(\mathrm{Re} / \mathrm{Rh})$ nuclei of the ventral midline thalamus abolished only the SWM-specific activity patterns that were seen during dual-task sessions within the hippocampal-prefrontal circuit. These findings demonstrate that $\mathrm{Re} / \mathrm{Rh}$ facilitate bidirectional communication between the dorsal hippocampus and mPFC during SWM, providing evidence for a causal role of $\mathrm{Re} / \mathrm{Rh}$ in regulating hippocampal-prefrontal synchrony and SWM-directed behavior.

Key words: dorsal hippocampus; hippocampal-prefrontal synchrony; medial prefrontal cortex; nucleus reuniens; spatial working memory

\section{Significance Statement}

Hippocampal-prefrontal synchrony has long been thought to be critical for spatial working memory (SWM) and the ventral midline thalamic reuniens and rhomboid nuclei $(\mathrm{Re} / \mathrm{Rh}$ ) have long been considered a potential site for synchronizing the hippocampus and medial prefrontal cortex. However, the hypothesis that Re/Rh are critical for hippocampal-prefrontal synchrony and SWM has not been tested. We first used a dual-task approach to identify SWM-specific patterns of hippocampal-prefrontal synchrony. We then demonstrated that Re/Rh inactivation concurrently disrupted SWM-specific behavior and the SWM-specific patterns of hippocampal-prefrontal synchrony seen during dual-task performance. These results provide the first direct evidence that Re/Rh contribute to SWM by modulating hippocampal-prefrontal synchrony.

\section{Introduction}

Rhythmic activity in neuronal populations creates oscillations in the brain's local field potential (LFP) (Buzsaki, 2006). Oscillatory

\footnotetext{
Received March 25, 2016; revised June 1, 2016; accepted June 15, 2016.

Author contributions: H.L.H. and A.L.G. designed research; H.L.H. performed research; H.L.H. and A.W. analyzed data; H.L.H. and A.L.G. wrote the paper.

This work is supported by the National Institute of Mental Health and by the National Institute of General Medical Sciences of the National Institutes of Health under grant numbers R01 MH102394 and P20 GM103650, respectively. We thank E. Myhre, B. Emanuel, and D. Layfield for behavioral assistance; M. Donahue, M. Patel, L. Moedinger, and A. Hallock for technical assistance; and A. Garcia and J. Neunuebel for comments on the manuscript.

The authors declare no competing financial interests.

Correspondence should be addressed to Amy L. Griffin, Department of Psychological and Brain Sciences, University of Delaware, 108 Wolf Hall, Newark, DE 19716. E-mail: amygriff@psych.udel.edu.

DOI:10.1523/JNEUROSCI.0991-16.2016

Copyright $\odot 2016$ the authors $\quad 0270-6474 / 16 / 368372-18 \$ 15.00 / 0$
}

synchrony between LFPs in disparate brain areas facilitates interregional communication, which is essential for encoding, consolidating, and retrieving memories (Fell and Axmacher, 2011). Phase synchronization between the prefrontal cortex (PFC) and medial temporal lobe is correlated with spatial working memory (SWM) in humans (Sarnthein et al., 1998; Serrien et al., 2004; Kopp et al., 2006; Payne and Kounios, 2009), rats (Jones and Wilson, 2005; Hyman et al., 2010), and mice (Sigurdsson et al., 2010; O'Neill et al., 2013; Spellman et al., 2015), suggesting that hippocampal-prefrontal synchrony is important for promoting SWM across mammalian species. In rodents, complementary lines of research have supported this hypothesis by showing that pharmacological disconnection of the medial PFC (mPFC) from the hippocampus results in SWM impairments (Lee and Kesner, 2003; Churchwell and Kesner, 2011). Decreases in hippocampal- 
prefrontal synchrony also parallel SWM deficits in both human schizophrenic patients (Lawrie et al., 2002; Meyer-Lindenberg et al., 2005) and a genetic mouse model of schizophrenia (Sigurdsson et al., 2010), highlighting the therapeutic importance of understanding how the hippocampus and PFC interact during SWM-guided behavior.

Although hippocampal-prefrontal interactions have been correlated with performance during SWM tasks in rodents (Jones and Wilson, 2005; Hyman et al., 2010; Sigurdsson et al., 2010; O'Neill et al., 2013; Spellman et al., 2015), the mechanisms that underlie this synchrony are not well understood. Lesions of the rodent dorsal hippocampus (Dudchenko et al., 2000; Ainge et al., 2007; Czerniawski et al., 2009; Hallock et al., 2013a), but not the ventral hippocampus (Czerniawski et al., 2009), disrupt SWM and the rodent mPFC functionally synchronizes with the dorsal hippocampus during successful performance of SWM tasks (Jones and Wilson, 2005; Hyman et al., 2010; Sigurdsson et al., 2010; O'Neill et al., 2013), supporting the conclusion that the rodent dorsal hippocampus is critical for SWM-guided decision making. Despite evidence that dorsal hippocampal-mPFC interactions are both correlated with (Jones and Wilson, 2005; Hyman et al., 2010; Sigurdsson et al., 2010) and necessary for (Lee and Kesner, 2003) SWM in rodents, the rodent $\mathrm{mPFC}$ receives direct inputs only from the ventral hippocampus (Swanson, 1981; Ferino et al., 1987; Jay et al., 1989; Thierry et al., 2000). How the dorsal hippocampus and mPFC communicate during SWM tasks, therefore, remains an open question. One potential mediator of dorsal hippocampal-mPFC synchrony is the ventral midline thalamus, which contains the reuniens (Re) and rhomboid $(\mathrm{Rh})$ nuclei. $\mathrm{Re} / \mathrm{Rh}$ are reciprocally connected with both the CA1 subfield of the dorsal hippocampus and the mPFC (Herkenham, 1979; Vertes et al., 2006; Vertes et al., 2007) and pharmacological inactivation of Re/Rh produces performance deficits in SWM tasks (Hembrook and Mair, 2011; Hembrook et al., 2012; Hallock et al., 2013b). These findings led us to hypothesize that $\mathrm{Re} / \mathrm{Rh}$ contribute to SWM by orchestrating hippocampal-prefrontal interactions (Griffin and Hallock, 2013). To uncover the mechanisms that regulate hippocampal-prefrontal synchrony during SWM, we performed two experiments. In Experiment 1, we compared mPFC population activity, oscillatory synchrony, and directionally specific interactions in the hippocampal-prefrontal circuit between an SWM-dependent delayed alternation (DA) task and an SWM-independent conditional discrimination (CD) task. We found that task representations are encoded within mPFC population activity only during successful SWM task performance. We further found that population coding in the mPFC was accompanied by SWM-specific patterns of directionally specific oscillatory synchrony between the dorsal hippocampus and mPFC. In Experiment 2, we show that pharmacological inactivation of Re/Rh concomitantly impairs SWM task performance, reduces hippocampal-prefrontal oscillatory synchrony, and abolishes directionally specific hippocampal-prefrontal interactions that were observed during the successful use of an SWMguided strategy in Experiment 1. Our findings provide direct evidence that $\mathrm{Re} / \mathrm{Rh}$ mediate SWM-guided decision making and hippocampal-prefrontal communication.

\section{Materials and Methods}

Subjects. Subjects were adult ( $>90 \mathrm{~d}$ old), male, Long-Evans hooded rats ( $n=5$ for Experiment $1 ; n=7$ for Experiment 2). Subjects weighed between 250 and $650 \mathrm{~g}$ at the time of surgery. During experiments, each subject was placed on mild food restriction ( $4-5$ food pellets per day) to keep each subject at $\sim 90 \%$ of his ad libitum body weight. The colony room was temperature and humidity controlled and each subject was maintained on a $12 \mathrm{~h}$ light/dark cycle. All experiments were performed during the light cycle.

Behavioral apparatus and testing room. The behavioral apparatus was a modified T-maze that was made of wood and painted black. The maze consisted of a central stem $(116 \times 10 \mathrm{~cm})$, two goal arms $(56.5 \times 10 \mathrm{~cm}$ each), and two return arms $(112 \times 10 \mathrm{~cm})$. Each section of the maze was surrounded by $6-\mathrm{cm}$-high wooden walls. Between trials, rats waited on a pedestal (a barstool with a ceramic dish attached to the top) that was located at the base of the T-maze. During intertrial intervals, the pedestal was occluded from the T-maze by a large wooden barricade that was placed between the pedestal and the maze stem. The T-maze was located in a room that was completely surrounded by black curtains. Several large visual cues were attached to the curtains (strips of red and green tape, patterned circles, and triangles). The room was dimly illuminated by a compact fluorescent bulb. Before and after recording sessions, rats were placed in a black plastic enclosure ( 8 inches tall, 14 inches in diameter) that was attached to the top of another stool. The bowl was lined with a thin sheet of cotton and placed in the corner of the testing room. Rats were placed in this bowl during attachment and detachment of the recording head stage and while tetrodes were being advanced into the $\mathrm{mPFC}$ and dorsal hippocampus. For Experiment 1, rats also waited in the enclosure during presession and postsession recording epochs.

Handling, pretraining, and task training. Handling and pretraining methods were identical to those described previously (Hallock and Griffin, 2013; Hallock et al., 2013a; Hallock et al., 2013b). Rats in Experiment 1 were first trained on a tactile-visual CD task. During this task, rats learned to make a behavioral response based on the texture and color of floor inserts that spanned the length of the stem and both goal arms. One side of the maze inserts was covered with black mesh and the other side was smooth wood (light brown). During each trial, either mesh or wood was presented according to a pseudorandom sequence (Fellows, 1967). Half of the rats were rewarded for turning right when mesh was present and left when wood was present; the other half learned the opposite rule (left on mesh, right on wood). The cue inserts were flipped between each trial even whether the same cue was presented on consecutive trials. This was done to ensure that the rat could not solve the task by using auditory cues. Between each trial, rats waited on the start box for $30 \mathrm{~s}$, at which time the experimenter prepared the inserts for the next trial and baited the appropriate goal zone. Rats were given one session of CD per day, with each session consisting of 24 trials. Once rats in Experiment 1 had reached a predetermined performance criterion of at least $80 \%$ correct choices (20/24 correct trials) for two consecutive sessions, a spatial DA task with a $30 \mathrm{~s}$ delay between trials was introduced into the training sessions. During the DA task, rats were rewarded for alternating between the left and right goal arms from trial to trial. Rats were given 24 trials of $\mathrm{CD}$ and then placed in their home cages for a period of $20 \mathrm{~min}$. After the 20 min period had ended, rats were trained on the DA task. Once rats learned to perform both the $\mathrm{CD}$ and DA tasks at a level of $80 \%$ correct choices (20/24 correct trials for both tasks), they underwent surgery for implantation of a recording microdrive.

For Experiment 2, rats were trained solely on the DA task and performed one session of DA per day, with each session consisting of 24 trials. Once rats reached a performance criterion of at least $80 \%$ correct choices (at least 20/24 correct) for two consecutive sessions, they underwent surgery for implantation of the recording microdrive and guide cannula.

Surgical procedures. Rats were anesthetized with isoflurane (1.0-3.0\% in oxygen), and placed into a stereotaxic instrument (Kopf). Puralube was applied to the rat's eyes and the incision site was subcutaneously injected with lidocaine and sterilized with Novalsan. An incision was made, the head was leveled, and bregma coordinates were identified. For rats in Experiment 1, four small bone screws (Fine Science Tools) were fitted into four small burr holes that were made with a stereotaxically mounted drill (Fine Science Tools). Dental acrylic (Lang Dental) was applied to anchor the screws to the skull. One circular hole was drilled above the mPFC ( $3.1 \mathrm{~mm}$ anterior to bregma, $0.5-2.0 \mathrm{~mm}$ from the midline, depending on angle of the microdrive bundle) and one hole was drilled above the dorsal hippocampus on the ipsilateral hemisphere (3.0 - 
$4.0 \mathrm{~mm}$ posterior to bregma, $1.5-3.0 \mathrm{~mm}$ from the midline, depending on angle of the microdrive bundle and distance from the mPFC bundle). A microdrive array with two tetrode-containing bundles (mPFC bundle: seven tetrodes; hippocampal bundle: 12 tetrodes for rats 1-4 and two tetrodes for rats 5-6) was lowered onto the surface of the brain and cemented to the skull with dental acrylic. For rats in Experiment 2, a circular hole was drilled $1.8 \mathrm{~mm}$ posterior to bregma and $2.0 \mathrm{~mm}$ lateral to the midline. An $8.0 \mathrm{~mm}$ guide cannula (Plastics One) targeting Re/Rh was lowered $6.5 \mathrm{~mm}$ ventral to the surface of the brain at a $15^{\circ}$ angle. Because Re/Rh are midline structures, only one cannula was implanted. The cannula was cemented to the skull with dental acrylic and a dummy cannula made to fit the guide cannula with a $1.0 \mathrm{~mm}$ projection was inserted. After the acrylic was dry, a dual-site microdrive targeting the mPFC and dorsal hippocampus was implanted using the same procedures detailed above for rats in Experiment 1. The guide cannula and microdrive were implanted in opposite hemispheres. For all experiments, the hemisphere of implantation was counterbalanced across rats. For both experiments, the microdrive was attached to a ground screw (a self-tapping bone screw with a piece of wire soldered to it), which was fitted into a small burr hole directly above the lambda skull suture. Each tetrode was then advanced $1.13 \mathrm{~mm}$ into the brain. All rats received a subcutaneous shot of flunixin (Banamine; $2.5 \mathrm{mg} / \mathrm{kg}$ ) and children's ibuprofen $(30 \mathrm{mg} / \mathrm{kg})$ in their drinking water for $2 \mathrm{~d}$ after surgery. Rats were allowed to recover for a period of at least $5 \mathrm{~d}$ after surgery before behavioral testing began. All procedures were approved by the University of Delaware Institutional Animal Care and Use Committee.

Recording sessions. For Experiment 1 recording sessions, neural activity from the $\mathrm{mPFC}$ and dorsal hippocampus was recorded for $1 \mathrm{~h}$ before task performance. Rats then performed an epoch of either DA or CD (task order was counterbalanced from session to session), followed by a $20 \mathrm{~min}$ period during which the rat remained plugged in and sat in the cottonlined black plastic bowl, followed by an epoch of either DA or CD (whichever task was not performed earlier). Rats 1 and 2 performed three task epochs per recording session (one epoch of DA, one epoch of CD, and another epoch of DA) with 20 min periods interleaved between each epoch. For analysis purposes, dual-task sessions were defined as recording periods during which the rat performed both a DA epoch and a CD epoch. Rats 1 and 2 therefore performed two dual-task sessions per recording period (first DA epoch vs $\mathrm{CD}$ epoch and $\mathrm{CD}$ epoch vs second DA epoch). After task performance, neural activity was recorded for another $1 \mathrm{~h}$ period. Presession and postsession recording periods were done to assess cluster stability during dual-task performance.

For Experiment 2 recording sessions, rats first performed a "baseline" epoch of DA (before any pharmacological manipulation) and then performed a "testing" epoch of DA (after pharmacological manipulation). Each rat underwent three session types in counterbalanced order; each session type consisted of a different pharmacological manipulation between baseline and testing epochs. For "no-infusion" sessions, rats were simply placed into their home cages for $30 \mathrm{~min}$ between epochs. For "saline" sessions, rats were given an infusion of PBS into Re/Rh and were allowed to sit in their home cages for $20 \mathrm{~min}$ between epochs. For "muscimol" sessions, rats were given an infusion of muscimol $\left(\mathrm{a} \mathrm{GABA}_{\mathrm{A}}\right.$ receptor agonist) into $\mathrm{Re} / \mathrm{Rh}$ and were placed in their home cages for 20 min between epochs.

Infusion protocol. For rats in Experiment 2, dummy cannulae were removed and an internal cannula made to fit the guide cannula with a 1.0 $\mathrm{mm}$ projection was inserted. Internal cannulae were attached to a tube that contained either PBS (Fisher Scientific) or muscimol (a GABA receptor agonist; Life Technologies) diluted to a concentration of 0.25 $\mu \mathrm{g} / \mu \mathrm{l}$ in PBS. The tube was attached to a microinfusion syringe (Hamilton) and placed into an automated infusion pump (World Precision Instruments) that controlled infusion rate and volume $(0.25 \mu \mathrm{l} / \mathrm{min}$ and $0.5 \mu \mathrm{l}$, respectively). Position of the infusate was monitored by marking an air bubble that separated the infusate from distilled $\mathrm{H}_{2} \mathrm{O}$ within the tubing. Internal cannulae sat in the brain for $2 \mathrm{~min}$ after infusion. Behavioral testing took place $30 \mathrm{~min}$ after infusions were given.

Perfusion and histology. Marking lesions were made by passing $10 \mu \mathrm{A}$ of current through one wire of each tetrode and reference electrodes. After $24 \mathrm{~h}$, rats were perfused with PBS, followed by $4 \%$ paraformalde- hyde, and the head was soaked in paraformaldehyde for 2-3 d. After raising the tetrodes out of the brain, the brain was removed from the skull and placed in a $9 \%$ sucrose solution. After sinking, the brains were frozen and sectioned $(40 \mu \mathrm{m})$.

Rats in Experiment 2 were given an infusion ( $0.5 \mu \mathrm{l}$ volume) of a fluorophore-conjugated muscimol (BODIPY TMR-X; Life Technologies) $30 \mathrm{~min}$ before perfusion (Allen et al., 2008). To visualize placement of the internal cannulae for rats in Experiment 2, half of the brain slices from $\mathrm{Re} / \mathrm{Rh}$ were stained using cresyl violet. The other half of the brain slices were stained with ProLong Gold with DAPI (Life Technologies) to visualize the spread of the fluorophore-conjugated muscimol. Brain slices containing tetrode tracks in the mPFC and dorsal hippocampus were mounted and stained with cresyl violet. DAPI-stained brain slices were visualized with a confocal microscope (LSM 710; Zeiss) and tiled fluorescent images were created using ZEN software (Zeiss). Cannula placement and tetrode tracks from all rats were visualized by placing digital plates from the Paxinos and Watson (2005) rat brain atlas over pictures of the cresyl-stained brain slices using Adobe Illustrator.

Statistical analysis. Specific statistical tests used are stated throughout the text. When distributions of dependent variables could not be assumed to be normal (i.e., firing rate distributions), nonparametric tests were used. Distributions of normalized scores were confirmed to be approximately Gaussian and were therefore analyzed with parametric tests (one-way ANOVAs for between-session type testing in Experiment 1; repeated-measures ANOVAs for within-rat testing in Experiment 2). Post hoc tests (pairwise comparisons) for all ANOVAs were Bonferroni corrected. An $\alpha$ level of 0.05 was used for all statistical tests.

Recording and cluster identification. Recordings were made with a 64channel digital recording system (Digital Lynx; Neuralynx). A ceilingmounted video camera captured position data $(30 \mathrm{~Hz})$ by recording luminance emitted by two LEDs attached to the rat's headstage. Cheetah software (Neuralynx) was used to visualize spikes from single units, as well as the continuously sampled LFP from the mPFC and dorsal hippocampus. LFP data were sampled at a rate of $2 \mathrm{kHz}$, and band-pass filtered between 1 and $600 \mathrm{~Hz}$. Spikes were sampled at $32 \mathrm{kHz}$, and band-pass filtered between $0.6-6 \mathrm{kHz}$. A spike waveform amplitude of $75 \mu \mathrm{V}$ was used as a threshold for single-unit spiking.

Individual clusters of spikes (putative individual neurons) were identified with KlustaKwik software, plotted with SpikeSort (Neuralynx) software, and manually selected based on differences in peak amplitude and energy between channels. Only clusters with an L-ratio $<0.1$ were included for subsequent analyses (Schmitzer-Torbert et al., 2005). Cluster stability was assessed by plotting the cluster's peak waveform over time and only clusters with a peak waveform that remained stable across the recording session were used. Putative interneurons were separated from putative pyramidal neurons based on differences in spike waveform width and interspike interval. Only putative pyramidal neurons were included in single unit and population analyses.

Analysis of $m P F C$ single units. Firing rates for each individual single unit recorded from the mPFC were calculated for three distinct sections of the T-maze: the start box (the pedestal on which rats waited between trials), the maze stem (the central portion of the maze), and the choice point (the T-intersection of the maze where the rat made a choice between the left or right goal arm). Firing rates were calculated by dividing the number of spikes emitted by that neuron by the amount of time that was spent at a particular maze section. For the 30-s-long start-box occupancy period, firing rate was first calculated for 1-s-long nonoverlapping sliding windows and then averaged across windows to produce a grand firing rate average. For Experiment 1, a 2 (task) $\times 3$ (maze section) mixed-factorial ANOVA with firing rate as the dependent variable was run for each recorded $\mathrm{mPFC}$ single unit to quantify task modulation of mPFC neuronal activity. A neuron was considered to be task modulated if either a significant section $\times$ task interaction or a significant betweenfactor main effect of task was observed. Task modulation was further assessed by assigning a discrimination index (DI) value to each recorded single unit as follows:

$$
\mathrm{DI}=a b s\left(F R_{\mathrm{DA}}-F R_{\mathrm{CD}}\right) /\left(F R_{\mathrm{DA}}+F R_{\mathrm{CD}}\right)
$$


where $F R_{\mathrm{DA}}$ is the mean firing rate during DA performance and $F R_{\mathrm{CD}}$ is the mean firing rate during $C D$ performance.

Linear classifier and receiver-operating characteristic (ROC) curves. To observe whether population firing rates in simultaneously recorded $\mathrm{mPFC}$ neurons could predict which task the rat was performing during Experiment 1, we tested a linear classifier on an array of mean firing rates from a simultaneously recorded population of $\mathrm{mPFC}$ neurons on a random trial of a recording session, trained the classifier on the remaining trials, and repeated this procedure until the classifier had been tested on all trials within the recording session. We used a support vector machine approach to construct a hyperplane in a high-dimensional subspace of mean firing rates based on the maximum margin between the hyperplane boundaries and each point of the training data. The classifier solves an optimization problem based on the training data as follows:

$$
\arg \min R(w)+C \sum_{i=1}^{N} L\left(y_{i}, w^{T} x_{i}\right)
$$

where $w$ are the parameters of the classifier, $R(\mathrm{w})$ is a term that prevents overfitting to the data, and $C$ is a penalty parameter for misclassification ( $C=1$ for all data in the current study). The output score of the classifier is defined by the following:

$$
y=f(\vec{w} \cdot \vec{x})=f\left(\sum_{j} w_{j} x_{j}\right)
$$

where $\vec{x}$ is a vector of mean firing rates, $\vec{w}$ is a vector of weights, and $f$ is a function that assigns a binary value $(1,-1)$ to the testing data. The output value of the classifier is then compared with its testing label $(1,-1)$ and the decoding accuracy of the classifier can be interpreted as the number of correctly identified trial types (either CD or DA) divided by the total number of trials in the recording session. The weights of the linear classifier were optimized based on the training data and the same weights were used for classification of the testing data. Recording sessions were categorized according to behavioral performance. Both correct and error trials were included in classifier training and testing sets. Classifier accuracy was further verified by calculating ROC curves for classifier outputs with the built-in MATLAB function perfcurve, with positive class labels set to 1 and negative class labels set to -1 .

Entrainment of mPFC single units to the hippocampal theta oscillation. Theta entrainment during intertrial intervals was calculated for each recorded single unit from the $\mathrm{mPFC}$ with custom MATLAB functions. Only clusters that contained at least 50 spikes that were able to be assigned a phase value were used for analysis. Spikes that occurred during bouts of low-amplitude theta oscillations were not assigned a phase value and were excluded from the analysis (see next section). Rayleigh's test of nonuniformity was used to test the null hypothesis that spikes are uniformly distributed across all theta phases. A Rayleigh's $z$-value was assigned to each neuron (with a uniform spike-phase distribution used as a null distribution) and a corresponding $p$-value was given. The mean resultant length (MRL) for each spike-phase distribution was then calculated, which indicates the direction (preferred phase) and magnitude of directionality (length) for the given distribution by first averaging direction vectors, calculating the preferred phase angle, and finally calculating the length of the mean resultant vector. A bootstrapped MRL and Rayleigh's $z$-value for each neuron was calculated by randomly subsampling 50 spike-phase pairs from the distribution 1000 times and taking the mean of the bootstrapped distribution. This was done to control for differences in number of spikes between neurons. Lag analysis was performed by shifting hippocampal LFPs in time and recalculating phase locking (MRL) at each temporal lag.

Power spectral density. For all LFP analyses, continuously sampled data were first detrended (i.e., low-frequency "drifting" artifacts were removed) with custom MATLAB functions. Raw signals were band-pass filtered with either a third-degree Butterworth filter or Morlet wavelet convolution for single-unit entrainment and phase-amplitude coupling analyses. For averaged spectral density estimation during start-box, stem, and choice-point occupancy, the multitaper method was used (Jarvis and Mitra, 2001). To exclude the possibility that "non-theta" states (i.e., periods when high-powered delta or ripple oscillations are present in the
LFP) influenced phase coherence and phase-amplitude coupling analyses during start-box occupancy, a theta/delta ratio was calculated by using a Hilbert transform to extract the instantaneous magnitude of theta $(4-12 \mathrm{~Hz})$ and delta $(1-4 \mathrm{~Hz})$ oscillations in the hippocampal LFP (Brandon et al., 2011). Periods of analysis during start-box occupancy were restricted to epochs during which the theta/delta ratio exceeded 4:1 (instantaneous theta amplitude was four times higher than instantaneous delta amplitude). Although LFPs recorded from the mPFC were generally lower in amplitude than those recorded from the dorsal hippocampus (especially in the delta and theta frequency bands), mPFC LFPs still contained prominent delta and theta oscillations. Band-pass filtering and spectral density estimation were performed in the same manner for both hippocampal and mPFC LFPs.

Phase coherence. Spectral coherence was estimated by using the multitaper method (Jarvis and Mitra, 2001). Chronux toolbox routines (function coherencyc.m) were used to estimate coherence for delta $(1-4 \mathrm{~Hz})$, theta $(4-12 \mathrm{~Hz})$, beta $(15-30 \mathrm{~Hz})$, and slow gamma $(30-80 \mathrm{~Hz})$ frequency bands. For time-frequency estimations, multitaper spectral coherence was calculated for $200 \mathrm{~ms}$ moving windows with 50 ms overlaps. Coherograms were used for visualization purposes only and were not compared statistically. Coherence values across maze segments were calculated with custom MATLAB functions.

Phase-amplitude coupling. To quantify phase-amplitude coupling, a modulation index (MI) value was calculated (Tort et al., 2010). Phase was extracted in the theta-frequency band by identifying peaks, troughs, and zero crossings and interpolating phase for each continuously sampled timestamp value; this method accounts for possible asymmetries within theta cycles (MATLAB code provided by M. Brandon). Gamma envelopes were extracted using a Hilbert transform. Each amplitude value of the gamma oscillation was then assigned a theta phase bin and amplitude was normalized by averaging across the number of observations for each phase bin, such that:

$$
P(j)=\frac{\left\langle A_{f_{A}}\right\rangle \varphi_{f_{p}}(j)}{\sum_{k=1}^{N}\left\langle A_{f_{A}}\right\rangle \varphi_{f_{p}}(k)}
$$

where $\varphi_{f_{p}}$ is equal to the time series for phases, $A_{f_{A}}$ is equal to the time series for amplitude envelope, and $N$ is equal to the number of bin observations (18 phase bins were used for all analyses in the current study). To quantify deviation of the observed phase-amplitude distribution from a uniform phase-amplitude distribution, the Kullback-Leibler distance was then calculated, which infers the amount of distance between two distributions. The MI value represents the difference between the observed phase-amplitude distribution and a uniform phase-amplitude distribution, with higher values indicating a larger deviation.

To create co-modulogram heat maps and polar plots of normalized gamma amplitudes in phase space, normalized amplitude values were calculated for each theta-phase bin across a range of gamma frequencies using custom MATLAB functions. For heat maps, instantaneous phase and amplitude for each frequency pair were extracted with Morlet wavelets. MI values across maze segments, frequency bands, and phases were calculated with custom MATLAB functions.

Granger causality. Bivariate Granger causality was used to predict changes in one LFP based on lagged values of the other LFP with custom MATLAB functions. For each LFP pair, a univariate autoregression was calculated for the mPFC LFP and augmented with lagged values of the hippocampal LFP. The optimal model order for each LFP pair was calculated by using the Bayes' information criterion. A Granger causality index (GCI) value was then assigned for each direction (GCI for hippocampal $\rightarrow$ prefrontal directionality and GCI for prefrontal $\rightarrow$ hippocampal directionality). For frequency-specific GCI values, Granger causality was first performed across the entire range of frequencies and GCI values across a predetermined frequency range (i.e., $30-80 \mathrm{~Hz}$ for slow gamma) were then averaged. To create a single directionality metric, lead indices (LIs) were calculated by dividing the GCI for hippocam$\mathrm{pal} \rightarrow$ prefrontal directionality by the total GCI for both directions. An LI value $>0.5$ indicated that the hippocampus led the mPFC, whereas a value $<0.5$ indicated that the $\mathrm{mPFC}$ led the hippocampus. 

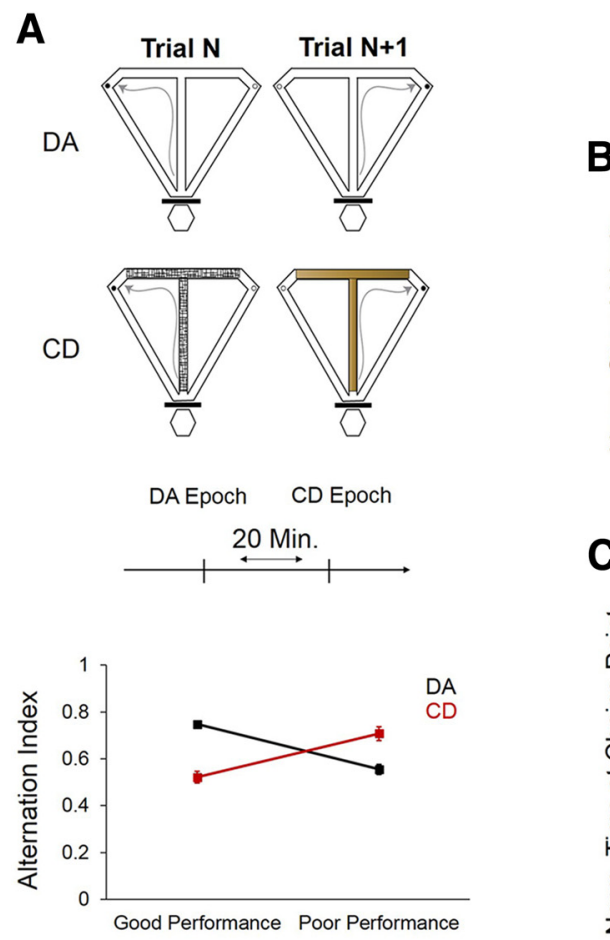

B

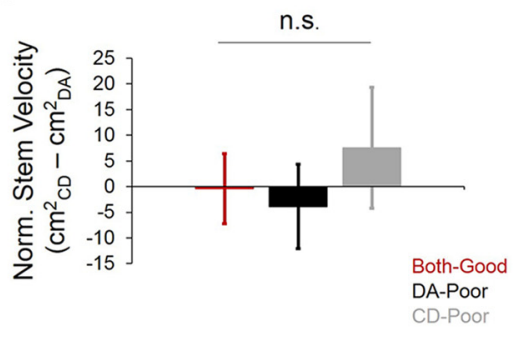

C

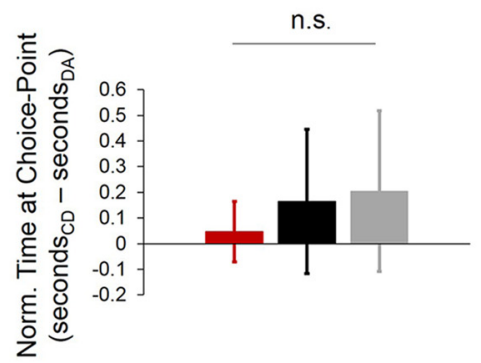

D
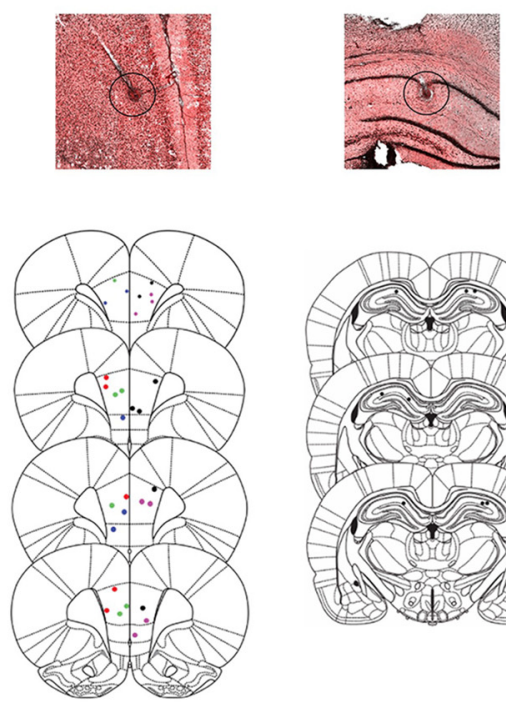

Figure 1. Behavior and histology from Experiment 1.A, Top, Schematic of the tasks used in Experiment 1. During DA, the rat is rewarded for alternating between the left and right goal arms on successive trials. Between trials, the rat waits on the start-box (hexagon at bottom of maze) for $30 \mathrm{~s}$. During CD, the appearance and texture of stem and goal-arm inserts predicts the location of reward. The rat also waits on the start-box for 30 s between trials of the CD task. Middle, Schematic of a "dual-task" recording session. Each dual-task session consists of one DA epoch,and one CD epoch. Between epochs, the rat waits in a bowl for $20 \mathrm{~min}$. The order in which task epochs were presented was counterbalanced across sessions. Bottom, Alternation index values during good-performance and poor-performance $D A$ and $C D$ epochs. An alternation index value of 1 indicates perfect alternation and an alternation index value of 0 indicates no alternation. Rats tended to alternate during good DA performance and poor CD performance compared with poor DA performance and good CD performance. $\boldsymbol{B}$, Normalized stem velocity was created by subtracting stem velocity during the $C D$ epoch from stem velocity during the $D A$ epoch. Therefore, if velocity dropped from $D A$ to $C D$, then the normalized velocity would be negative; if it increased from $D A$ to $C D$, then the normalized velocity would be positive. If there was no change, then the normalized velocity would be zero. Normalized stem velocity was not significantly different between the three dual-task session types (both-good, DA-poor, and CD-poor). C, Normalized time spent at the choice point did not significantly differ between session types. D, Histological verification of tetrode lesions in the mPFC and dorsal hippocampus of rats in Experiment 1. Top two panels (left, mPFC; right, dorsal hippocampus) show example histology from one rat, with tetrode lesions outlined by black circles. Bottom panels show locations of tetrode lesions from rats in Experiment 1 (colors $=$ individual rats). For $\boldsymbol{B}$ and $\boldsymbol{C}$, error bars indicate $95 \%$ confidence intervals for 1 -sample $t$ tests with 0 null and n.s. $=$ no significant main effect of session type for one-way ANOVA $(p>0.05)$.

\section{Results}

\section{Experiment 1}

Behavior

To observe SWM-specific patterns of neural activity in the dorsal hippocampus and $\mathrm{mPFC}$, we trained one group of rats to perform both an SWM-independent CD and SWM-dependent DA task (Fig. $1 A$ ) within a behavioral session; therefore, each "dual-task" session consisted of one DA and one CD epoch (Fig. 1A). Although rats generally performed both tasks well, there were some dual-task sessions in which behavioral performance on either task was below asymptote, allowing us to further compare neural activity and behavior between "good" ( $>75 \%$ choice accuracy) and "poor" ( $<75 \%$ choice accuracy) task epochs ( $75 \%$ threshold chosen based on $p<0.05$ for binomial probability test). We suspected that poor performance on the CD task epochs was due to the incorrect utilization of an alternation strategy. Therefore, to quantify the degree to which rats alternated between goal arms during task performance, we calculated an alternation index by dividing the number of trials during which rats alternated (visited the opposite goal arm from the previous trial) by the total number of trials. Therefore, perfect alternation would result in an alternation index value of 1 , whereas visiting the same goal arm on every trial would result in an alternation index value of 0 . A 2 (task) $\times 2$ (performance) univariate ANOVA revealed a significant task by performance interaction $\left(F_{(1,75)}=89.573, p<\right.$
0.001), indicating that rats tended to alternate between goal arms during poor CD performance and good DA performance compared with good CD performance and poor DA performance (Fig. 1A).

Our dual-task approach allowed us to record the same single units and LFPs from the same animal within each behavioral session (between task epochs). To assess within-session (between-epoch) changes in behavior and neural activity, we further assigned each dual-task session to 1 of 3 categories for behavioral, single-unit, population, and LFP analyses based on whether the rat performed both tasks well or one of the tasks poorly (both good sessions, $n=24$; DA-poor sessions, $n=14$; CD-poor sessions, $n=9$ ). This approach allowed us to characterize whether within-rat behavior and neural activity differed as a function of task and if these differences could be attributed to variations in mnemonic strategy (SWM vs non-SWM). Behavior and neural activity was then normalized to a single value for each session type by subtracting the value during DA performance from the value during $\mathrm{CD}$ performance. Therefore, if the value was lower during $\mathrm{CD}$ than during $\mathrm{DA}$, then the normalized value would be negative; conversely, if the value was higher during $C D$ than during DA, then the normalized value would be positive. Finally, if there was no difference between values during DA and during $\mathrm{CD}$, the normalized value would be zero. In both the DA and CD tasks, the outcome of a single trial (correct or incorrect) 
A
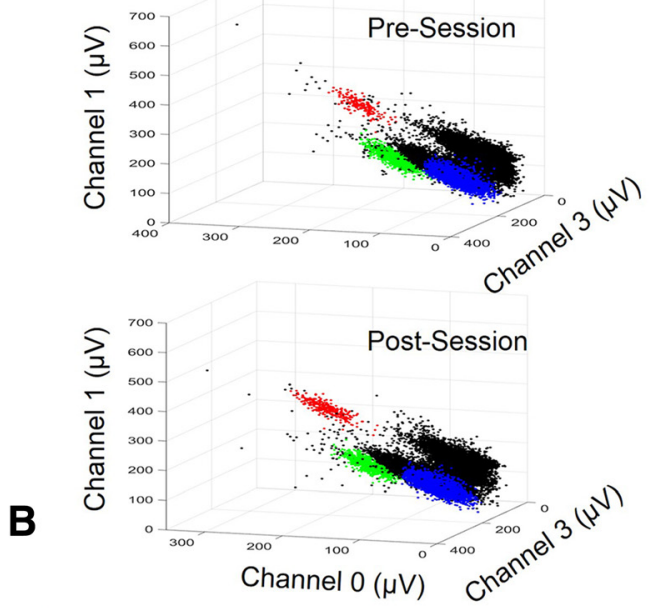

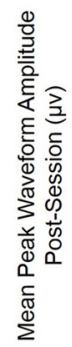

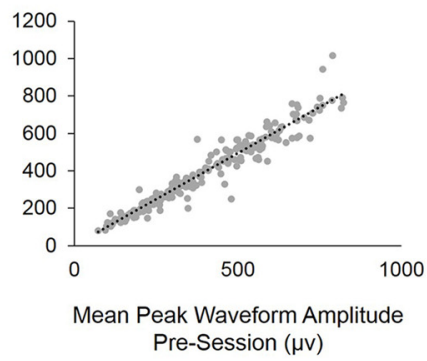

C

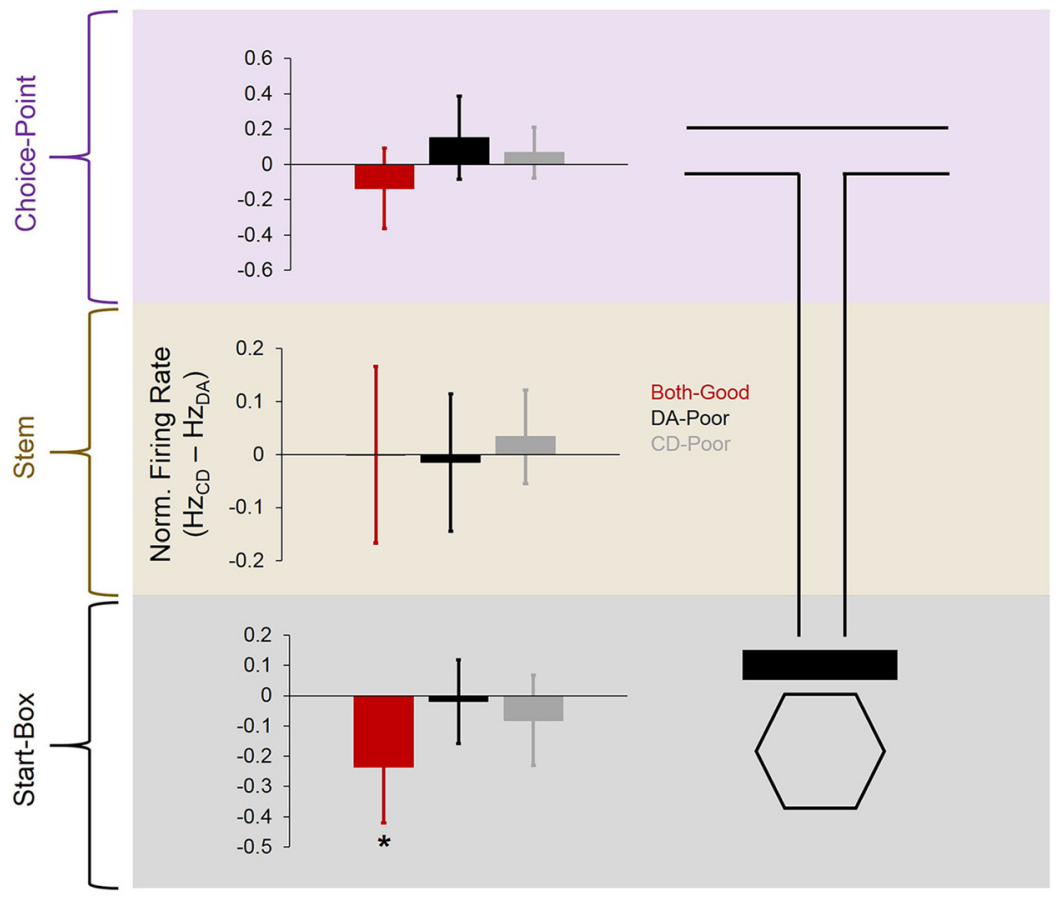

Figure 2. Firing rates of single units recorded from the $\mathrm{mPFC}$ during dual-task sessions. $\boldsymbol{A}$, Example of three clusters recorded simultaneously from one tetrode in the mPFC of one rat during a dual-task session. Clusters remained stable between presession and postsession recordings, indicating that any observed differences in firing rate between tasks was not a function of cluster instability. $\boldsymbol{B}$, Peak waveform amplitudes of all mPFC single units during presession and postsession recordings were highly and significantly correlated. $\boldsymbol{C}$, Normalized firing rates of mPFC neurons did not differ significantly between session types at any maze location; however, the normalized firing rate was significantly lower than zero during both-good sessions during start-box occupancy. Error bars indicate $95 \%$ confidence intervals for 1 -sample $t$ tests with 0 null, ${ }^{*} p<0.05$ for 1 -sample $t$ test with 0 null.

could be due to usage of the correct rule (a true correct trial) or it could occur by chance (e.g., even if the rat is not actively using an SWM-guided strategy during a DA trial, there is a $50 \%$ chance that he could nevertheless visit the correct goal arm). We therefore reasoned that comparing behavior and neural activity between session types, rather than between individual correct and incorrect trials, would give us a better indication of whether dependent variables differed as a function of SWM. Although task performance (percentage of correct choices) differed between the three session types, overt behavior did not because neither normalized (CD-DA) stem velocity (both-good: $t_{(23)}=-0.112, p=$ 0.91 , DA-poor: $t_{(13)}=-1.019, p=0.33$, CD-poor: $t_{(9)}=1.456$, $p=0.18$; Fig. $1 C$ ) nor the amount of time spent at the choice point (both-good: $t_{(23)}=0.81, p=0.42$, DA-poor: $t_{(13)}=1.269$, $p=0.23$, CD-poor: $t_{(9)}=1.478, p=0.17$; Fig. $\left.1 D\right)$ differed significantly from zero for any session type (1-sample $t$ tests with 0 null). The amount of time that each rat spent in the start box was restricted to $30 \mathrm{~s}$ for both tasks.

\section{Histology}

The majority of tetrode lesions (27/30) in postmortem brain slices of the mPFC were localized to the prelimbic (PL) subregion. The remaining three tetrode lesions were located in the infralimbic (IL) subregion. The limited number of tetrode lesions in the IL precluded a quantitative analysis of subregional differences in single-unit analyses, but no qualitative differences in firing rate or task modulation were observed between single units recorded from tetrodes putatively located in the IL or PL. All tetrode lesions in the hippocampus were localized to dorsal CA1; most lesions appeared to be in stratum lacunosum moleculare and this was further evidenced by high-amplitude theta oscillations and positively deflecting sharp waves that were present in the hippocampal LFP from the majority of hippocampal tetrodes (see Fig. $1 E$ for tetrode lesion placements). During recordings, the hippocampal tetrodes with the highest-amplitude theta oscillations were selected for LFP analyses.

\section{Single-unit analyses}

A total of 210 well isolated single units (putative pyramidal neurons) were recorded from the $\mathrm{mPFC}$ from three rats (rats 4 and 5 did not have single units and were therefore used solely for LFP analyses). To ensure cluster stability across tasks, we recorded for $1 \mathrm{~h}$ both before and after dual-task sessions. Mean peak waveform amplitude recorded from the tetrode channel with the highest peak waveform was calculated for each cluster and compared between presession and postsession recording epochs (Fig. 2A). Presession peak waveform amplitude was nearly perfectly correlated with postsession peak waveform amplitude when all single units were included $\left(\beta=12.1, p=4.3 \times 10^{-8}\right.$, Poisson regression), indicating that any observed differences in firing rate between tasks was not a function of cluster or tetrode instability (Fig. 2B). To assess firing rate differences between tasks, we first calculated a within-neuron normalized firing rate score by subtracting each neuron's mean firing rate during DA epochs from its mean firing rate during $\mathrm{CD}$ epochs. If a neuron had a higher firing rate during $C D$ epochs, then its normalized firing rate value would be positive; conversely, if it had a higher firing rate during DA epochs, then its normalized firing rate value would be nega- 
A

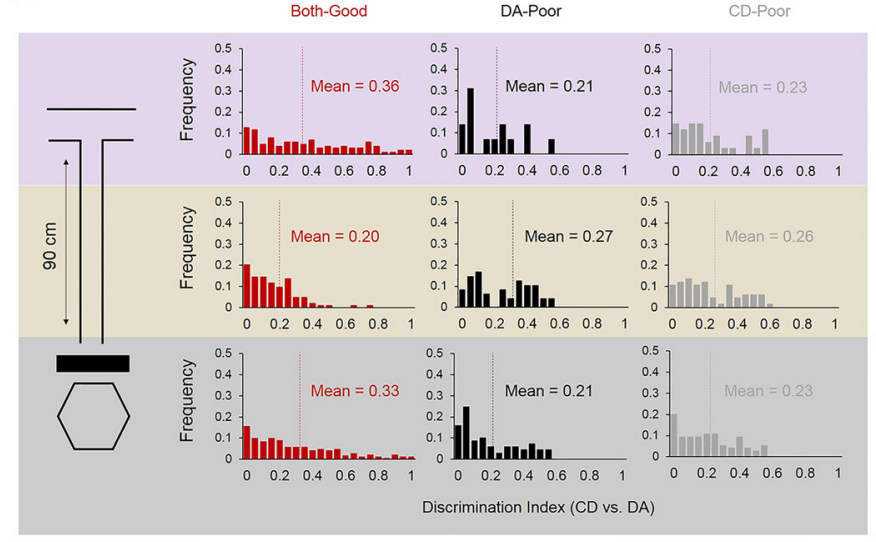

B

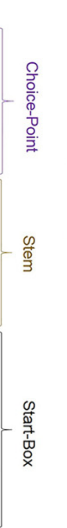

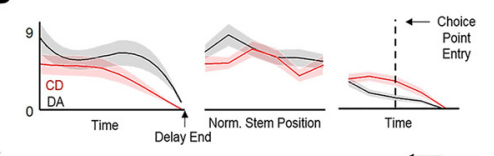
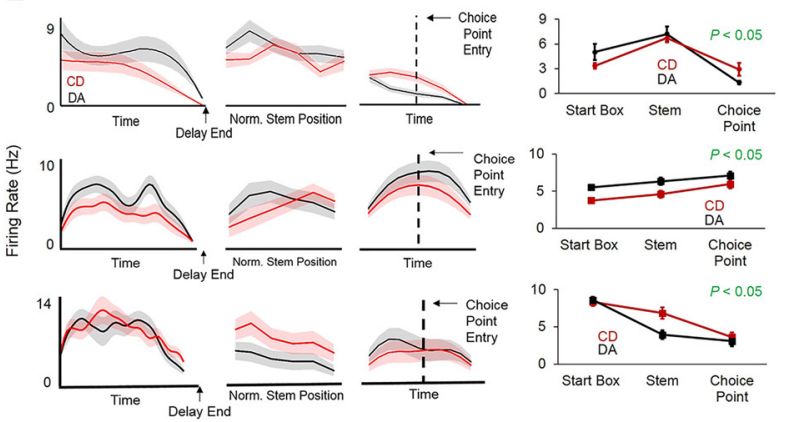

Cask-Modulated
Non Task-Modulated
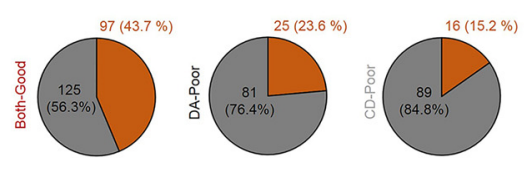

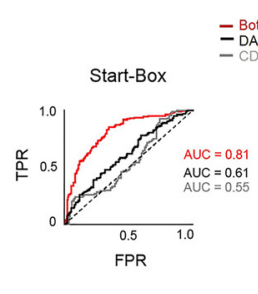

Both-Good
- DA-Poor
CD-POor
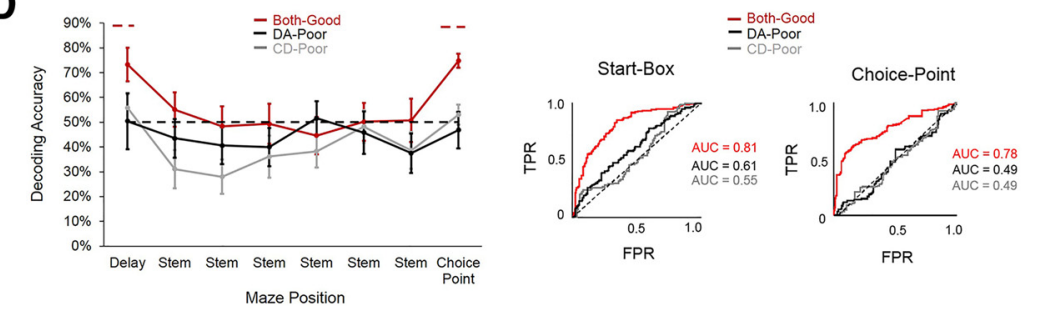

Figure 3. mPFC neurons distinguish between tasks during both-good sessions. A, DI scores for all mPFC single units recorded during dual-task sessions. A higher DI score indicates a higher firing rate difference between tasks. Means of the DI distributions are highest during both choice-point traversals and start-box occupancy of both-good sessions. $\boldsymbol{B}$, Examples of three mPFC neurons that were task modulated. These neurons showed a firing rate difference between the DA and CD tasks (left three panels = raw firing rate plots for start-box occupancy, stem traversals, and choice-point traversals; thick lines $=$ mean firing rate, shaded areas $=S E M)$, as evidenced by a significant task $\times$ maze location interaction with firing rate as the dependent variable $($ far right panels; markers $=$ means, error bars = SEM). $\boldsymbol{C}$, The highest proportion of task-modulated neurons was observed during both-good sessions. $\boldsymbol{D}$, Top, A linear classifier can correctly predict which task the rat is performing based on the aggregate activity of simultaneously recorded mPFC neurons at levels significantly above chance during start-box occupancy and choice-point traversals of both-good sessions. Error bars indicate SEM, dashed red lines indicate maze locations at which decoding accuracy during both-good sessions was significantly above chance $(p<0.05$ for binomial probability test). Bottom, ROC curves for classifier performance across session types. During both start-box occupancy and choice-point traversals, AUC is significantly $>0.5$ (chance). TPR, True-positive rate (proportion of correctly identified DA trials); FPR, false-positive rate (1 - proportion of correctly identified CD trials).

tive. Although normalized firing rates did not significantly differ between session types $\left(F_{(2,224)}=2.275, p=0.11\right.$, one-way ANOVA), we found that normalized firing rate scores were significantly lower than zero during start-box occupancy only when the rat performed both the DA and CD tasks well (both-good sessions: $\left.t_{(103)}=-2.527, p=0.01\right)$, but not when the rat performed either task poorly (DA-poor: $t_{(88)}=-.218, p=0.78$, CD-poor: $\left.t_{(110)}=-1.097, p=0.28\right)$. Normalized firing rates on the maze stem $\left(t_{(117)}=-0.293, p=0.77\right)$ and maze choice point $\left(t_{(117)}=-1.179, p=0.24\right)$ were not significantly different from zero for good-performance sessions (Fig. 2C). These results suggest that $\mathrm{mPFC}$ single-unit firing rates are, on average, higher during start-box occupancy in good DA performance epochs compared with start-box occupancy in good CD performance epochs. Normalized firing rates are indicative of changes in firing rate that occur in one direction (i.e., lower firing rates during $\mathrm{CD}$ than during DA); however, they do not capture differences in firing rate that may vary in direction between neurons. To further characterize task-related changes in firing rate, we calculated a DI score (see Materials and Methods) for each single unit, which reflects the net firing rate difference (whether positive or negative) between task epochs (higher DI values equal larger net firing rate differences). As expected, DI score distributions were significantly different between sessions during start-box occupancy $\left(H_{(2)}=9.1, p=0.01\right.$, Kruskal-Wallis test). During stem traversals, DI scores did not significantly differ between session types $\left(H_{(2)}=0.62, p=0.76\right)$; however, DI scores did significantly differ between session types during choice-point traversals $\left(H_{(2)}=\right.$
8.83, $p=0.01$, Kruskal-Wallis test), indicating that withinneuron differences in firing rate between the DA and CD tasks were also tied to behavioral performance when the rat was making his goal-arm decision (Fig. $3 A$ ). These results suggest that task representations in mPFC single units become more diverse and less stereotyped during choice-point traversals compared with during start-box occupancy. Finally, we assessed task modulation for each single unit by quantifying between-task differences in firing rate across all maze sections. A single unit was considered to be task modulated if either a significant task $X$ maze section interaction or significant between-task main effect of firing rate was found for a 2 (task) $\times 3$ (maze section) mixed-factorial ANOVA (Fig. 3B). A large proportion of single units recorded during both-good sessions were significantly task modulated (97/ $222,43.7 \%$ ), whereas a smaller proportion were task modulated during DA-poor $(16 / 105,15.2 \%)$ and CD-poor $(25 / 106,23.6 \%)$ sessions (Fig. 3C).

\section{Linear classifier}

Many mPFC single units differentiated between tasks; however, these neurons did not differentiate between tasks in the same way (i.e., firing rate differences were not consistently in one direction between neurons). This led us to hypothesize that task representations in the mPFC may ultimately be coded in a highdimensional subspace, rather than at the level of individual units. To probe task coding at the population level, we investigated whether summed activity patterns in $\mathrm{mPFC}$ neuronal ensembles could predict which task was being performed. We trained a lin- 
A

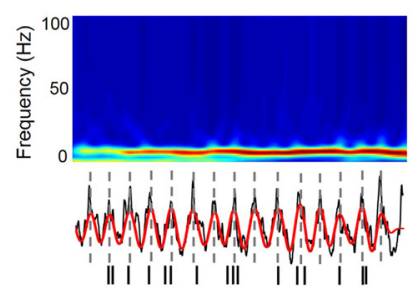

B

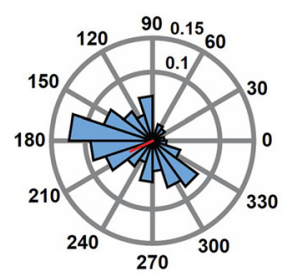

C

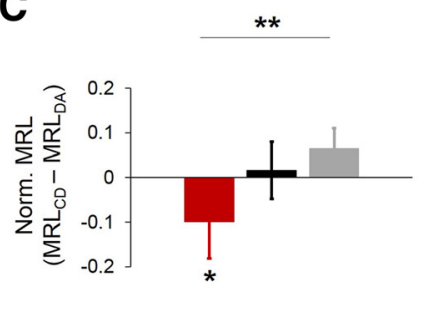

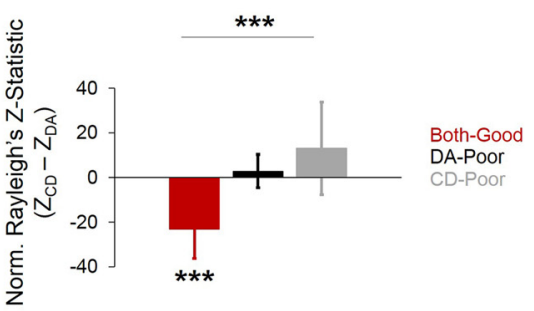

Figure 4. $\mathrm{mPFC}$ single-unit entrainment to hippocampal theta oscillations during start-box occupancy of dual-task sessions. $\boldsymbol{A}$, Example schematic of mPFC single-unit entrainment to the dorsal hippocampal theta oscillation during start-box occupancy of a good DA epoch. Top, High-amplitude theta oscillations in the hippocampal LFP. Middle, Raw hippocampal LFP in gray, with filtered theta oscillations superimposed in red. Bottom, Spikes emitted by a simultaneously recorded mPFC single unit (one tick mark = one spike). Dashed gray lines demonstrate that spikes from this neuron tended to occur around the peaks of the simultaneously recorded hippocampal theta oscillation. $\boldsymbol{B}$, Rose plot with hippocampal theta phase (degrees) on the circumference, proportion of spikes emitted in blue histogram bins, and MRL in red from a representative mPFC neuron recorded during start-box occupancy of a DA epoch. Note the nonuniform spike-phase distribution, indicating that spikes tended to occur around the $200^{\circ}$ phase of the simultaneously recorded dorsal hippocampal theta oscillation. $C$, Left, Normalized MRL scores were significantly different between session types and normalized MRL scores during both-good sessions were significantly <0. Right, As in left, but for normalized Rayleigh's z-statistic scores. Error bars indicate means and $95 \%$ confidence intervals for 1-sample $t$ tests with 0 null. Bars with asterisks above them indicate a significant main effect of session type for a one-way ANOVA. Asterisks below individual bars indicate a significant deviation from 0 for a 1 -sample $t$ test. ${ }^{*} p<0.05,{ }^{* *} p<0.01,{ }^{* * *} p<0.001$.

ear classifier with the mean firing rates of simultaneously recorded mPFC single units across maze bins, trained the classifier to distinguish between the CD and DA tasks, and categorized the classifier's decoding accuracy according to the session category (both-good, DA-poor, CD-poor). Decoding accuracy was significantly above chance for both-good sessions during start-box occupancy and choice-point traversals $(n$ trials correctly decoded $=$ 289 and 297/396, respectively; $p<0.05$, binomial probability tests) and did not significantly differ from chance when the rat performed either task poorly ( $p>0.05$ for all spatial bins, binomial probability tests; number of single units $=7.9 \pm 3.3,7 \pm 3.7$ and $8.5 \pm 5.1$, means $\pm \mathrm{SD}$, for both-good, DA-poor, and CDpoor respectively; Fig. $3 D$ ). To further verify classifier accuracy at the start box and choice point, ROC curves were created for classifier output at each maze location for both-good, DA-poor, and CD-poor sessions. Area underneath the ROC curve (AUC) was significantly different from 0.5 (nondiscrimination line) during start-box occupancy $\left(t_{(23)}=4.037, p<0.001\right)$ and choicepoint traversals $\left(t_{(23)}=5.607, p<0.001 ; 1\right.$-sample $t$ tests for AUC with 0.5 null) only for both-good sessions (DA-poor start-box: $t_{(13)}=1.535, p=0.15$, choice-point: $t_{(13)}=-1.187, p=0.26$, CD-poor start-box: $t_{(9)}=0.146, p=0.89$, choice-point: $t_{(9)}=$ $-0.2, p=0.85$; Fig. $3 D$ ). These results demonstrate that taskspecific representations are encoded within the aggregate activity of $\mathrm{mPFC}$ populations and are further tied to successful SWMguided behavior.

\section{Single-unit entrainment}

We next compared the extent to which $\mathrm{mPFC}$ neurons entrained to hippocampal theta oscillations between the SWM-dependent DA task and the SWM-independent CD task. Of 210 total mPFC neurons recorded during dual-task sessions, 137 (65.2\%) emitted $>50$ spikes during start-box occupancy of both DA and CD, allowing us to compare entrainment within the same neuron across the two tasks directly. During both-good sessions, $37.7 \%$ of single units included in entrainment analyses showed significant ( $p<0.05$ for Rayleigh's test of nonuniformity) entrainment during the DA epoch, but not the CD epoch. In contrast, only $7 \%$ showed the same pattern during DA-poor sessions and only $9.1 \%$ showed the same pattern during CD-poor sessions. To control for possible differences in spike count between tasks, subsampled phase-locking values were extracted from bootstrapped spikephase distributions for each neuron (see Materials and Methods). To quantify within-neuron changes in entrainment, we calcu- lated normalized Rayleigh's $z$-scores $\left(Z_{\mathrm{CD}}-Z_{\mathrm{DA}}\right)$. If entrainment were higher during $\mathrm{DA}$ than during $\mathrm{CD}$, then the normalized score would be negative; if entrainment were lower during DA than during CD, then the normalized score would be positive. Normalized $z$-scores were significantly different between session types $\left(F_{(2,136)}=7.499, p=0.001\right.$, one-way ANOVA) and normalized $z$-scores were significantly lower than zero $\left(t_{(72)}=-3.554, p=0.001,1\right.$-sample $t$ test with 0 null) for both-good sessions, but not DA-poor or CD-poor sessions $(p>$ 0.05; Fig. 4B). Normalized scores were also created for MRLs, another metric of phase-locking $\left(\mathrm{MRL}_{\mathrm{CD}}-\mathrm{MRL}_{\mathrm{DA}}\right)$. Results for normalized MRL scores paralleled those for normalized Rayleigh's $z$-scores, with normalized MRL scores being significantly different between session types $\left(F_{(2,136)}=5.874, p=0.004\right.$, oneway ANOVA) and lower than zero only for both-good sessions $\left(t_{(72)}=-2.401, p=0.02\right)$, but not DA-poor or CD-poor sessions $(p>0.05)$, demonstrating that strong $\mathrm{mPFC}$ unit theta entrainment during start-box occupancy is tied to successful SWMguided task performance (Fig. $4 B$ ). During CD performance in CD-poor sessions, Rayleigh's $z$-statistic and MRL values were higher, on average, than during CD performance in DA-poor and both-good sessions. This phenomenon could be tied to the incorrect usage of an SWM-guided strategy (alternation) during poor CD performance (Fig. 1A).

\section{Phase coherence}

To investigate hippocampal-prefrontal phase coherence during SWM-guided behavior, we simultaneously recorded LFPs from the dorsal hippocampus and mPFC during dual-task performance. Raw phase coherence scores were calculated for 4 distinct frequency bands (delta, $0-4 \mathrm{~Hz}$; theta, $4-12 \mathrm{~Hz}$; beta, $15-30 \mathrm{~Hz}$; slow gamma, $30-80 \mathrm{~Hz}$ ) and compared at three maze sections (start-box, stem, and choice-point). Normalized phase coherence scores were created by subtracting coherence values during DA from coherence values during CD. Normalized theta (4-12 $\mathrm{Hz}$ ) coherence scores were significantly different between session types $\left(F_{(2,48)}=4.465, p=0.02\right.$, one-way ANOVA $)$ and lower than zero $\left(t_{(23)}=-5.197, p<0.001\right)$ during choice-point traversals of both-good sessions, but not choice-point traversals of DA-poor $\left(t_{(13)}=0.35, p=0.73\right)$ or CD-poor $\left(t_{(9)}=-0.731, p=\right.$ 0.48 , 1-sample $t$ tests with 0 null) sessions (Fig. $5 A$ ). Choicepoint-normalized coherence scores were not significantly different between session types for any other frequency band $\left(\right.$ delta: $F_{(2,48)}=0.191, p=0.83$, beta: $F_{(2,48)}=2.515, p=0.1$, 
A

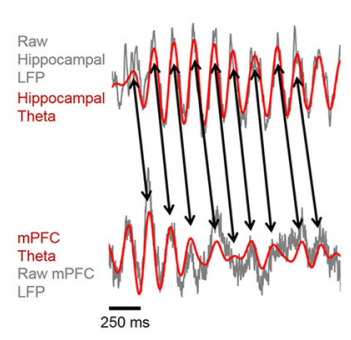

B

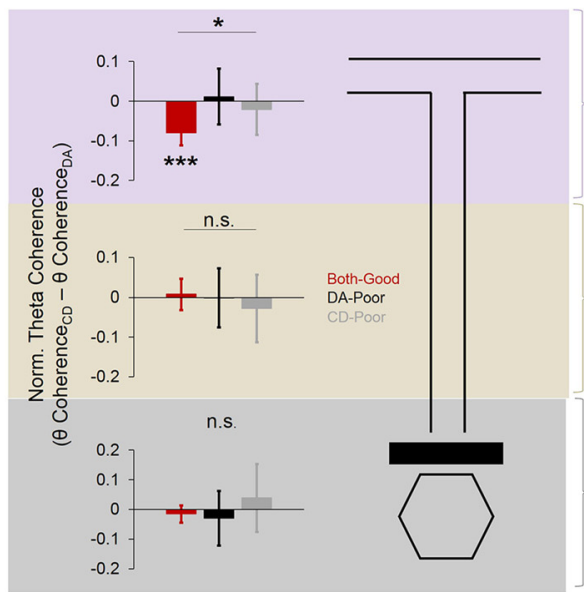

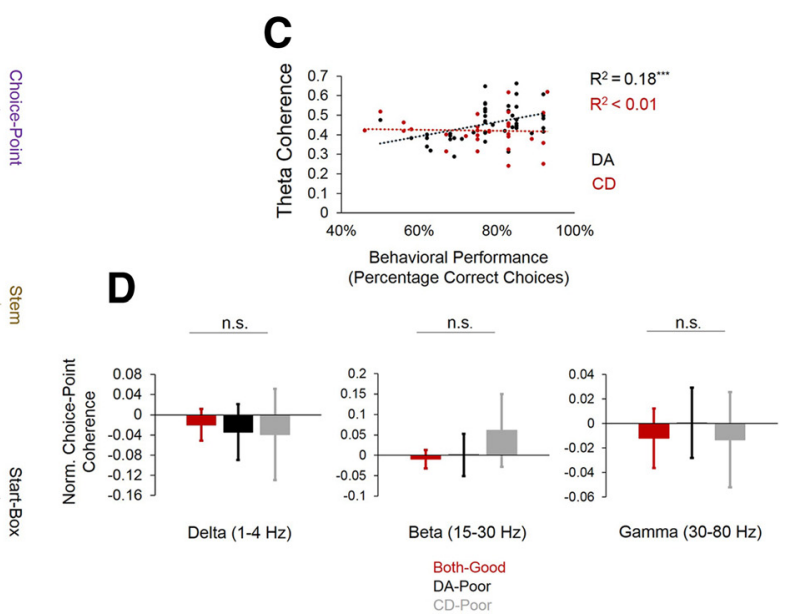

Figure 5. Theta-phase coherence during choice-point traversals differs as a function of dual-task performance. $A$, Example schematic of theta-phase coherence during a single choice-point traversal of a good DA epoch. Raw LFP traces are shown in gray and filtered theta oscillations are superimposed in red (top, dorsal hippocampal LFP; bottom, mPFC LFP). Black arrows show that the phases of the two oscillations are temporally coherent between cycles. $\boldsymbol{B}$, Normalized theta-phase coherence is significantly different between session types during choice-point traversals (top), but not stem traversals (middle) or start-box occupancy (bottom). C, Theta-phase coherence during choice-point traversals is significantly correlated with behavioral performance during DA epochs (black dots), but not CD epochs (red dots). D, Choice-point coherence in other frequency bands does not significantly differ between session types (left, delta coherence; middle, beta coherence; right, slow-gamma coherence). For $\boldsymbol{B}$ and $\boldsymbol{D}$, error bars means and $95 \%$ confidence intervals for 1 -sample $t$ tests with 0 null. Bars with asterisks above them indicate a significant main effect of session type for a one-way ANOVA. Asterisks below individual bars indicate a significant deviation from 0 for a 1-sample $t$ test. ${ }^{*} p<0.05,{ }^{* * *} p<0.001$, n.s. $=$ no significant main effect of session type for one-way ANOVA.

A

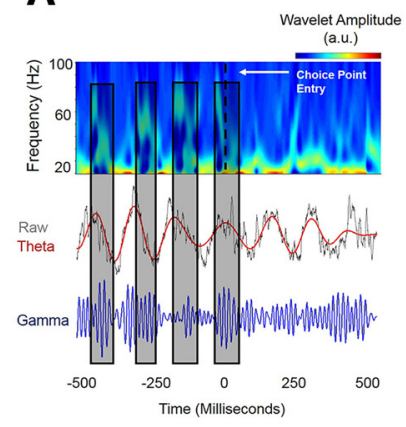

B

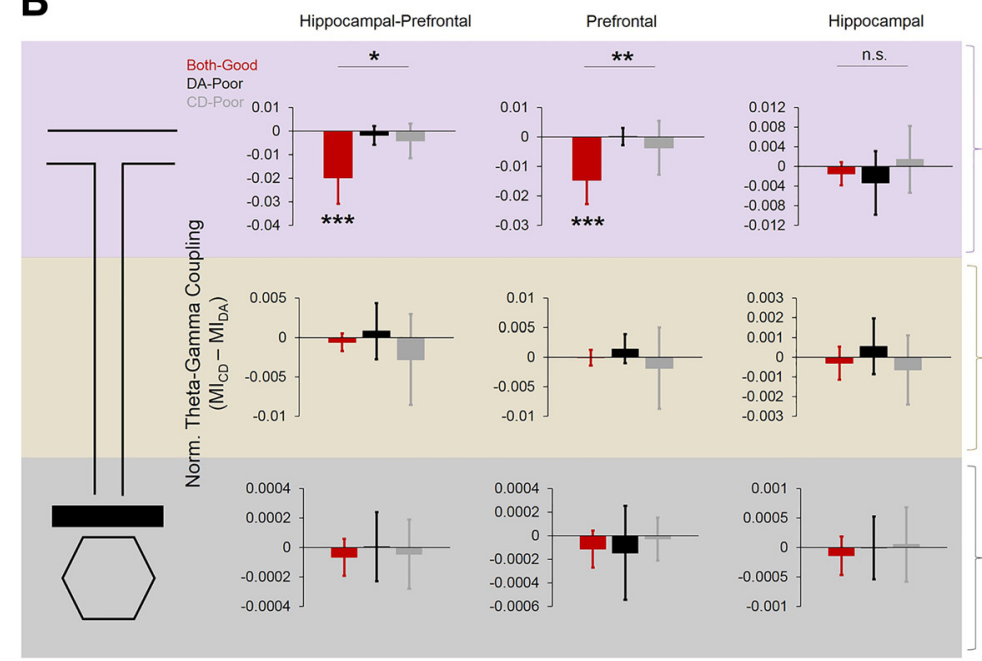

C
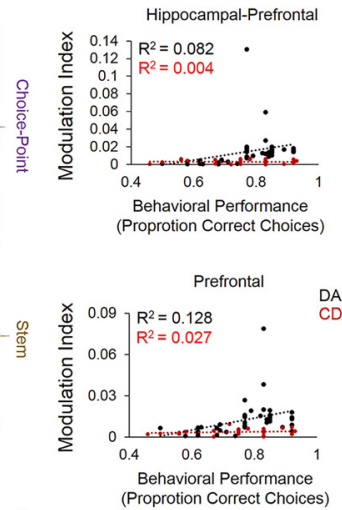

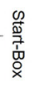

Figure 6. Theta-gamma phase-amplitude coupling during choice-point traversals differs as a function of task performance during dual-task sessions. $A$, Example schematic of hippocampal theta-prefrontal gamma-phase amplitude coupling during a single choice-point traversal of a good DA epoch. Top, Spectrogram of the prefrontal LFP. Middle panel, raw hippocampal LFP (gray) and superimposed theta oscillations (red). Bottom, filtered slow-gamma oscillations from the prefrontal LFP (blue). Bouts of high-amplitude slow gamma in the prefrontal LFP tend to cluster around the descending phase of the simultaneously recorded hippocampal theta oscillation (black rectangles). $\boldsymbol{B}$, Normalized theta-gamma phase-amplitude coupling during choice-point traversals (top three panels) differs significantly between session types. Choice-point theta-gamma coupling differences were observed for theta-gamma coupling between the dorsal hippocampus and mPFC (left) and within the MPFC (middle), but not within the dorsal hippocampus (right). Theta-gamma coupling between the hippocampus and mPFC, within the mPFC, or within the hippocampus did not significantly differ between session types during stem traversals (middle) or start-box occupancy (bottom). Error bars indicate means and $95 \%$ confidence intervals for 1 -sample $t$ tests with 0 null. Bars with asterisks above them indicate a significant main effect of session type for a one-way ANOVA. Asterisks below individual bars indicate a significant deviation from 0 for a 1-sample $t$ test. ${ }^{*} p<0.05,{ }^{* *} p<0.01,{ }^{* * *} p<0.001$, n.s. $=$ no significant main effect of session type for one-way ANOVA. C, Hippocampal-prefrontal theta-gamma coupling during choice-point traversals was significantly correlated with behavioral performance during DA epochs, but not CD epochs (top). Prefrontal theta-gamma coupling during choice-point traversals was also significantly correlated with behavioral performance during DA epochs, but not $C D$ epochs (bottom).

gamma: $F_{(2,48)}=0.276, p=0.76$, one-way ANOVAs; Fig. $5 C$ ). Differences in theta-phase coherence were restricted to the maze choice-point because normalized theta coherence did not differ significantly between session types for stem traversals $\left(F_{(2,48)}=\right.$ $0.372, p=0.69)$ or start-box occupancy $\left(F_{(2,48)}=1.108, p=0.34\right.$, one-way ANOVAs; Fig. 5A). Choice-point theta coherence was also significantly correlated with choice accuracy during DA $\left(R^{2}=0.183, p=0.001\right)$, but not CD $\left(R^{2}=0.002, p=0.42\right.$, linear regressions; Fig. $5 B$ ). Choice-point coherence in other frequency bands was neither correlated with DA choice accuracy (delta; $R^{2}=0.001, p=0.4$, beta; $R^{2}=0.02, p=0.17$, gamma; $R^{2}<$ $0.001, p=0.49)$ nor CD choice accuracy (delta; $R^{2}<0.001$, 
A MUUWUWUWUWUWU $\mathrm{Y} \rightarrow \mathrm{Y}(30-80 \mathrm{~Hz})$
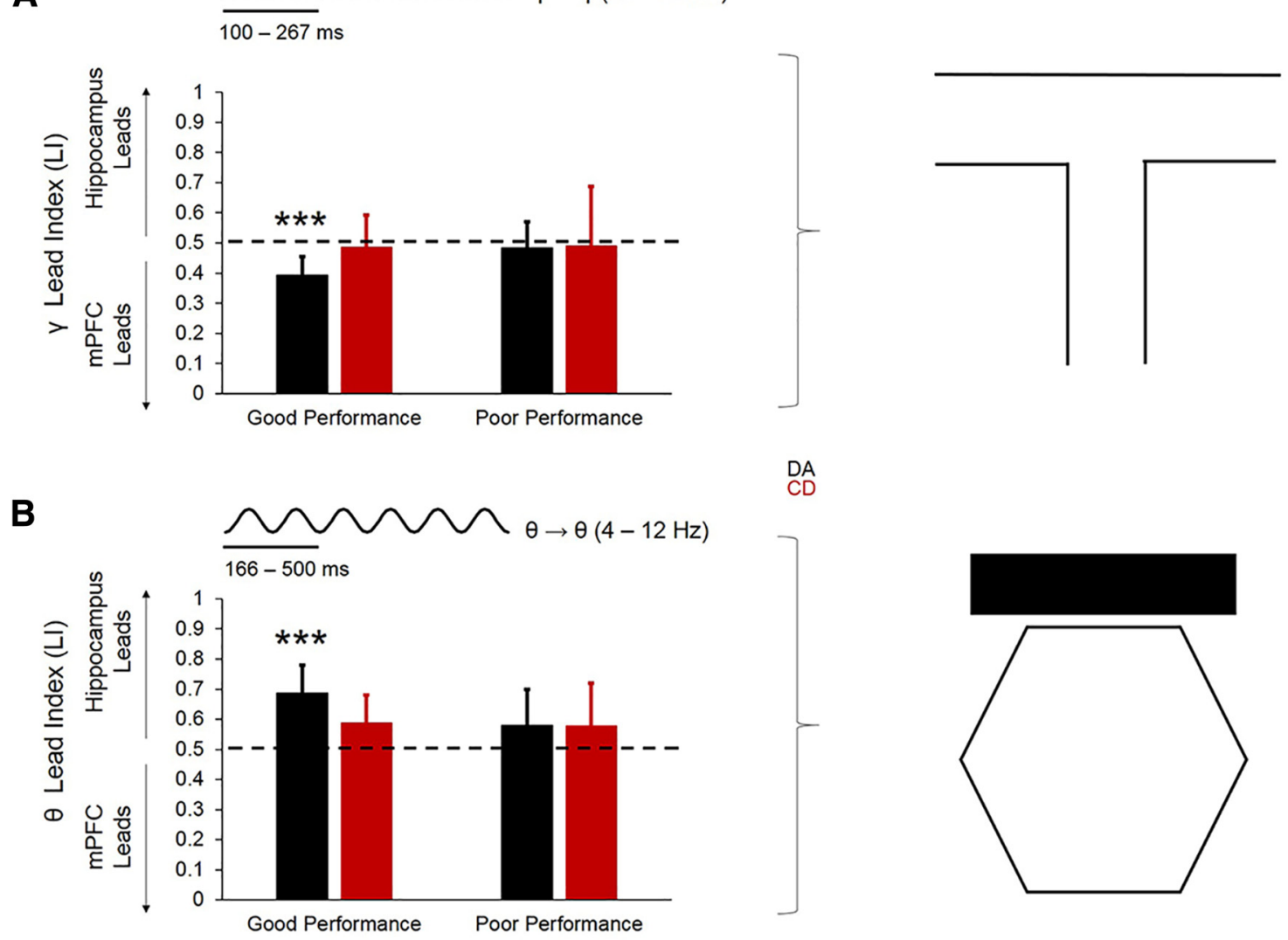

DA
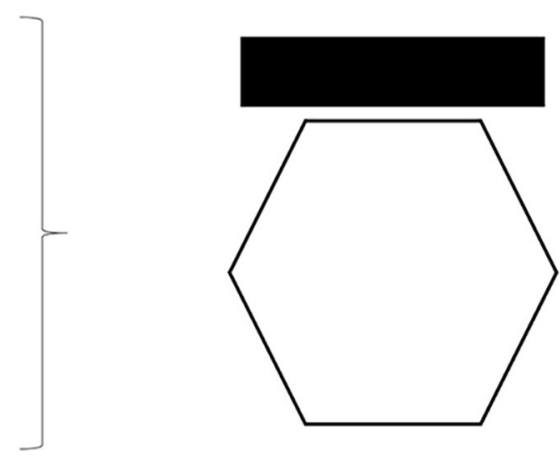

Figure 7. Directionality in the hippocampal-prefrontal circuit differs as a function of maze location and behavioral performance during dual-task sessions. $\boldsymbol{A}$, During choice-point traversals of good DA epochs, the mPFC led the dorsal hippocampus in the slow-gamma-frequency band. Error bars indicate means and $95 \%$ confidence intervals for 1-sample $t$ tests with 0.5 null. $\boldsymbol{B}$, During start-box occupancy of good DA epochs, the dorsal hippocampus led the mPFC in the theta-frequency band. Error bars indicate means and $95 \%$ confidence intervals for 1 -sample $t$ tests with 0.5 null. Asterisks above individual bars indicate a significant deviation from 0.5 for a 1-sample $t$ test. ${ }^{* *} p<0.001$.

$p=0.5$, beta; $R^{2}=0.03, p=0.18$, gamma; $R^{2}=0.002, p=0.42$, linear regressions; data not shown). Differences in theta power could not explain differences in theta-phase coherence because normalized theta power was not significantly different between sessions in either brain region (hippocampus: $F_{(2,48)}=0.476, p=$ 0.62 , mPFC: $F_{(2,48)}=1.784, p=0.18$, one-way ANOVAs).

\section{Phase-amplitude coupling}

We next performed phase-amplitude coupling analyses between the hippocampus and the mPFC and within each structure, comparing across session types. We found that, at the choice-point, phase-amplitude coupling between theta oscillations and slowgamma oscillations $(30-80 \mathrm{~Hz})$ differed significantly between session types. This was the case for phase-amplitude coupling both between hippocampal theta oscillations and mPFC gamma oscillations and theta-gamma coupling within the mPFC. Normalized MI scores $\left(\mathrm{CD}_{\mathrm{MI}}-\mathrm{DA}_{\mathrm{MI}}\right)$ for hippocampal-prefrontal theta-gamma coupling were significantly different between session types $\left(F_{(2,48)}=4.476, p=0.02\right.$, one-way ANOVA $)$ and lower than zero for both-good sessions $\left(t_{(23)}=-3.683, p=0.001\right)$, but not DA-poor $\left(t_{(13)}=-1.004, p=0.33\right)$ or CD-poor $\left(t_{(9)}=\right.$ $-1.309, p=0.22$ ) sessions (Fig. $6 A$ ). The same pattern of results was observed for normalized MI scores for prefrontal thetagamma coupling $\left(F_{(2,48)}=4.705, p=0.01\right.$, one-way ANOVA: both-good: $t_{(23)}=-3.728, p=0.001$, DA-poor: $t_{(13)}=0.059$, $p=0.95$, CD-poor: $t_{(9)}=-0.918, p=0.38,1$-sample $t$ tests with 0 null; Fig. $6 A$ ). Normalized MI scores were not significantly different between session types for hippocampal theta-gamma coupling $\left(F_{(2,48)}=0.964, p=0.39\right.$, one-way ANOVA; Fig. $\left.6 A\right)$. Consistent with the theta-phase coherence results described above, both hippocampal-prefrontal theta-gamma coupling $\left(R^{2}=0.08, p=0.03\right)$ and mPFC theta-gamma coupling $\left(R^{2}=\right.$ $0.128, p=0.007)$ at the maze choice point were significantly correlated with DA performance, but not CD performance (hippocampal-prefrontal: $R^{2}=0.004, p=0.37$, prefrontal: $R^{2}=$ $0.027, p=0.19$, linear regressions; Fig. $6 B$ ). Differences in thetagamma coupling were specific to the choice point because no differences in normalized MI were seen for any session type in any brain region on both the stem (hippocampal-prefrontal: $F_{(2,48)}=1.396, p=0.26$, prefrontal: $F_{(2,48)}=2.19, p=0.12$, hippocampal: $F_{(2,48)}=1.398, p=0.26$ ) and start box (hippocampal-prefrontal: $F_{(2,48)}=0.202, p=0.82$, prefrontal: $F_{(2,48)}=$ $1.608, p=0.21$, hippocampal: $F_{(2,48)}=0.428, p=0.66$, one-way ANOVAs; Fig. 6A).

\section{Granger causality}

To establish whether communication between the dorsal hippocampus and mPFC occurs in a directionally specific manner, we used Granger causality to calculate an LI (see Materials and Methods), with scores $>0.5$ indicating that the hippocampal LFP led the prefrontal LFP and scores $<0.5$ indicating that the prefrontal LFP led the hippocampal LFP. During choice-point traversals, the mPFC led the dorsal hippocampus in the slowgamma $(30-80 \mathrm{~Hz})$ frequency band selectively during good DA performance. Choice-point LI scores for slow gamma (but not delta, theta, or beta) were significantly $<0.5$ during good DA 
A

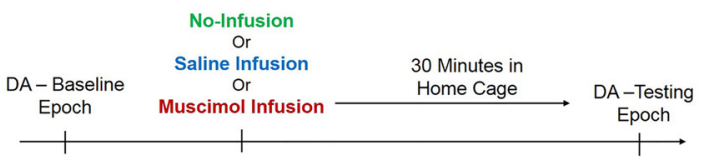

B
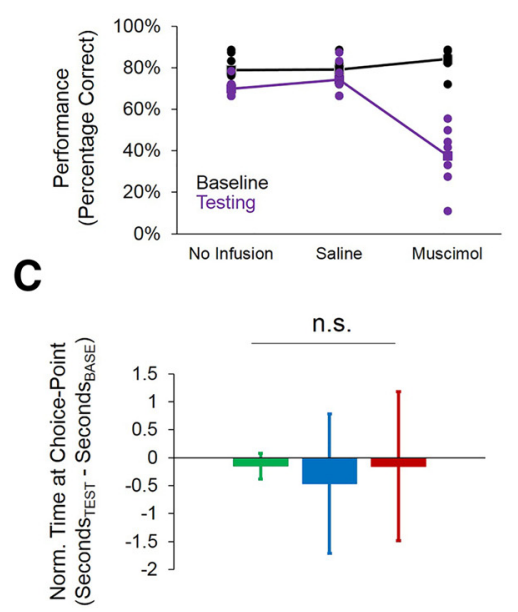
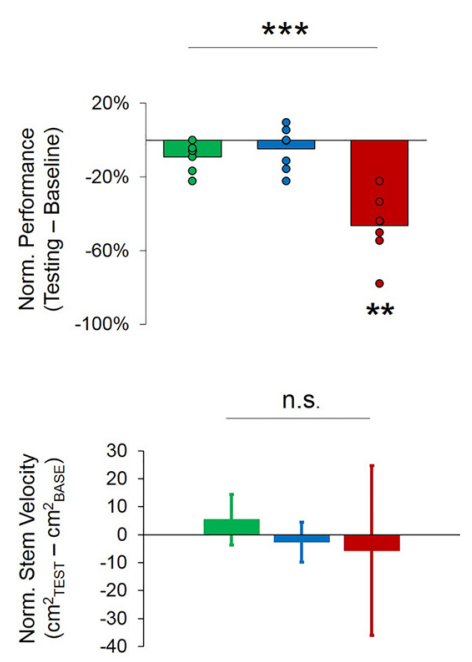

D
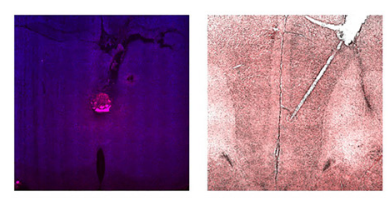
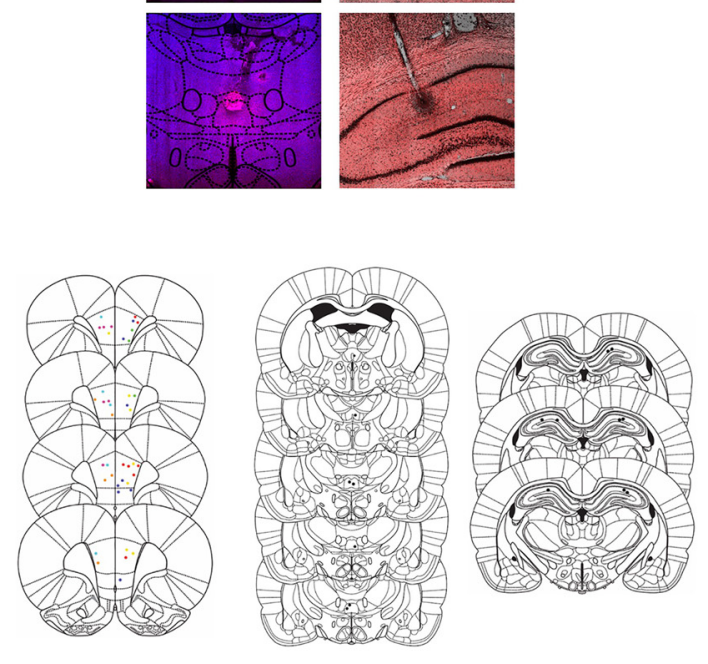

Figure 8. Behavior and histology for Experiment 2. A, Schematic of recording sessions for Experiment 2. Rats first performed a baseline (premanipulation) epoch of DA, followed by no infusion, a saline infusion, or a muscimol infusion into Re/Rh (session order counterbalanced across rats). After the infusion, rats performed a second testing (postmanipulation) epoch of DA. Behavior and neural activity could therefore be compared between baseline and testing epochs to assess differences that occurred as a result of pharmacological manipulation of Re/Rh. $B$, Muscimol infusions into Re/Rh significantly impaired DA performance. Left, Raw performance scores across infusion conditions (lines indicate means; dots indicate individual rats). Right, Normalized (testing - baseline) performance scores across infusion conditions (bars indicate means; dots indicate individual rats). C, Neither time spent at the choice point nor stem velocity significantly differed between infusion conditions. Error bars indicate means and $95 \%$ confidence intervals for 1-sample $t$ tests with 0 null. $\boldsymbol{D}$, Histological verification of cannula placement and muscimol spread in Re/Rh, as well as tetrode lesions in the mPFC and dorsal hippocampus for Experiment 2. Top panels show example histology from one rat. Left two panels show spread of a fluorophore-conjugated muscimol in Re/Rh. Right two panels show tetrode lesions in the MPFC (top) and dorsal hippocampus (bottom). Bottom panels show placements of tetrode lesions in the mPFC (left), cannula placements in Re/Rh (middle), and tetrode lesions in the dorsal hippocampus (right) from all rats included in Experiment 2.

performance $\left(t_{(30)}=-3.569, p=0.001,1\right.$-sample $t$ test with 0.5 null), but not poor DA performance $\left(t_{(13)}=-0.294, p=0.77\right)$, good CD performance $\left(t_{(18)}=-0.383, p=0.71\right)$, or poor CD performance $\left(t_{(7)}=1.294, p=0.24\right.$; Fig. $\left.7 A\right)$.

During start-box occupancy, LI in the theta (but not delta, beta, or slow gamma) frequency band during good DA performance was significantly $>0.5\left(t_{(30)}=4.049, p<0.001,1\right.$-sample $t$ test), whereas LI for theta during poor DA performance $\left(t_{(13)}=\right.$ $2.069, p=0.06)$, good CD performance $\left(t_{(18)}=1.399, p=0.18\right)$, or poor CD performance $\left(t_{(7)}=1.294, p=0.24\right)$ did not significantly differ from 0.5 (Fig. $7 B$ ). These data suggest that the direction of information flow between the hippocampus and mPFC depends on the maze location (and presumably on distinct cognitive operations that are required at that location), such that the hippocampus transmits information to the mPFC in the theta-frequency band during start-box occupancy, whereas the mPFC transmits information to the hippocampus in the gammafrequency band during choice-point traversals.

\section{Experiment 2 \\ Behavior}

To observe the impact of $\mathrm{Re} / \mathrm{Rh}$ inactivation on behavior and neural activity, we trained a second group of rats on the DA task and pharmacologically inactivated $\mathrm{Re} / \mathrm{Rh}$ with muscimol while simultaneously recording from the dorsal hippocampus and $\mathrm{mPFC}$ during DA performance. Rats took part in three recording sessions, each of which used a different infusion condition (no infusion, saline, or muscimol). During each session, rats performed a preinfusion baseline epoch and a postinfusion testing epoch of DA (Fig. 8A). Normalized performance scores were created by subtracting the percentage of correct choices during baseline epochs from the percentage of correct choices during testing epochs. Re/Rh inactivation significantly impaired DA performance $\left(F_{(2,12)}=25.173, p<0.001\right.$, main effect of infusion condition, repeated-measures ANOVA; Fig. $8 B$ ). Normalized performance scores during the muscimol condition were significantly lower than normalized performance scores during both the no-infusion $(p=0.009)$ and saline conditions $(p=$ 0.003; Bonferroni-corrected pairwise comparisons for repeatedmeasures ANOVAs). In most cases, Re/Rh inactivation nearly abolished alternation behavior, which, in our free-choice DA paradigm, would result in performance scores that are $<50 \%$ correct choices. Changes in overt behavior could not account for differences in choice accuracy between task epochs during dualtask performance or after muscimol infusions because neither stem velocity nor time spent at the maze choice point differed significantly as a function of infusion condition (no infusion, saline, and muscimol; $F_{(2,12)}=0.508, p=0.61$ for normalized velocity, $F_{(2,12)}=0.273, p=0.77$ for normalized time spent at choice point, repeated-measures ANOVAs; Fig. $8 C$ ).

\section{Histology}

Of eight rats that were implanted with a cannula targeting $\mathrm{Re} / \mathrm{Rh}$ and a microdrive targeting the $\mathrm{mPFC}$ and dorsal hippocampus, seven had cannulae that terminated in either Re or Rh (the eighth rat developed an infection surrounding the cannula tip, so the location of the cannula tip could not be verified). We used fluorescent muscimol to verify that infusions remained restricted to 


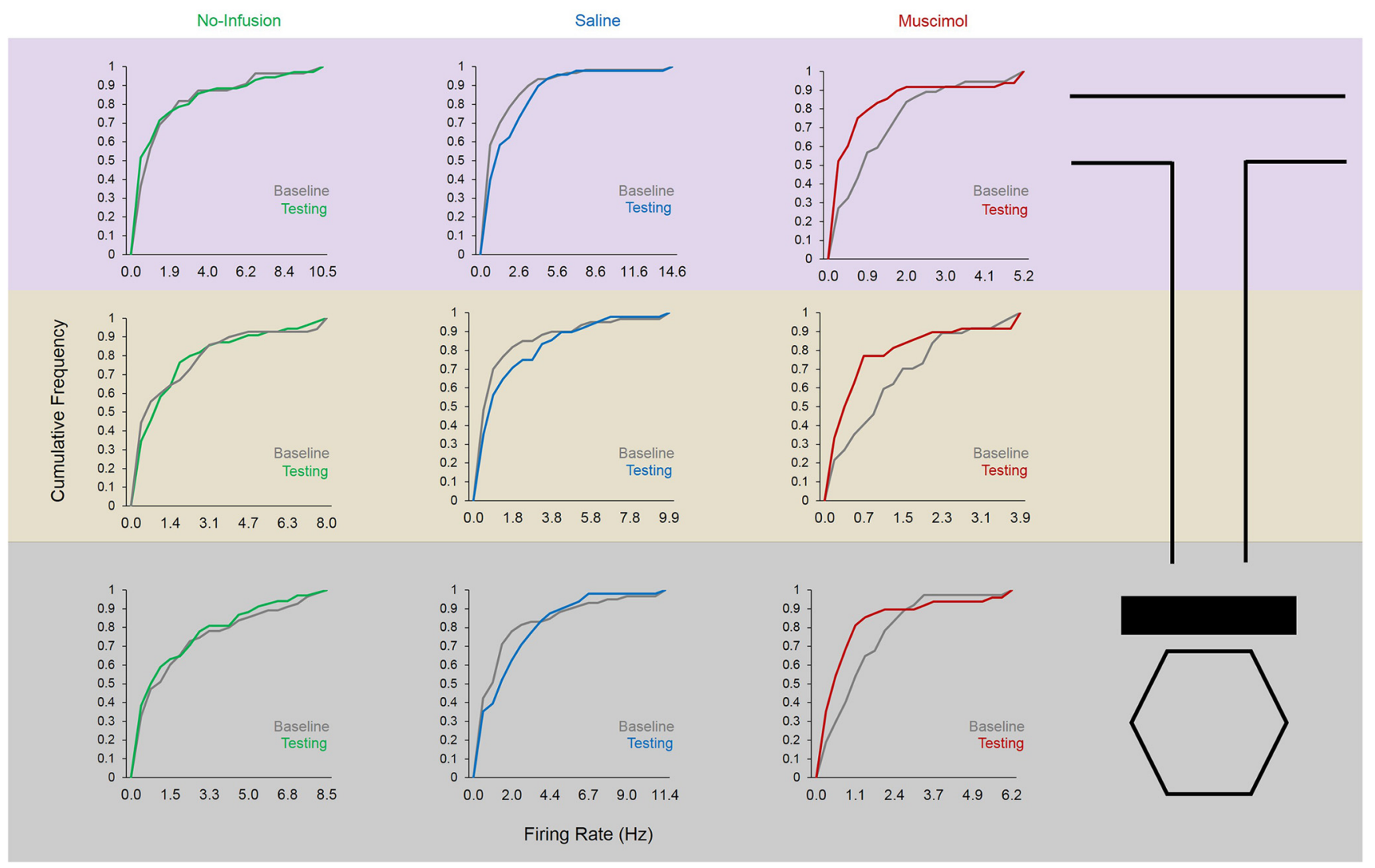

Figure 9. mPFC single-unit firing rates are decreased after Re/Rh inactivation. Muscimol infusions into Re/Rh significantly decrease firing rates in the mPFC at all maze locations (choice-point, stem, and start-box). Columns indicate infusion condition; rows indicate maze location, and cumulative density plots detail the cumulative distributions of firing rate for mPFC single units recorded during each epoch of each infusion condition.

$\mathrm{Re} / \mathrm{Rh}$. In five of seven rats, the fluorescent signal remained entirely restricted to Re/Rh. In the remaining two rats, the fluorescent signal was largely confined to Re/Rh, but minimal fluorescence was observed in the adjacent submedius thalamic nucleus. Even though fluorescence was not observed at further distances from the injection site, it is possible that the concentration of muscimol that was infused was nevertheless sufficient to activate $\mathrm{GABA}_{\mathrm{A}}$ receptors beyond the boundaries of the ventral midline thalamus. Behavioral performance and neural activity did not differ qualitatively between the five rats that had fluorescent signaling restricted to Re/Rh and the two rats that had minimal fluorescent signaling in the submedius thalamic nucleus.

As with rats in Experiment 1, the vast majority of tetrode lesions were observed in the PL subregion of the MPFC (38/41 lesions), whereas the remaining three lesions were observed in the IL subregion. All hippocampal tetrode lesions were located in dorsal CA1 below the stratum pyramidale (Fig. $8 D$ ).

\section{Single-unit entrainment}

A total of 270 well isolated single units (putative pyramidal neurons) were recorded from the mPFC of six rats in Experiment 2 (single units were not able to be recorded from rat 7 , which was used solely for behavioral and LFP analyses). Re/Rh inactivation significantly decreased $\mathrm{mPFC}$ single unit firing rates at all sections of the maze (muscimol baseline vs testing, start box: $p=0.003$, stem: $p=0.003$, choice-point: $p=0.004$, Wilcoxon's rank-sum tests; Fig. 9). These data suggest that muscimol infusions into $\mathrm{Re} / \mathrm{Rh}$ decreased population firing rates in the anatomically connected $\mathrm{mPFC}$, possibly by reducing excitatory drive from $\mathrm{mPFC}-$ projecting cells in $\mathrm{Re} / \mathrm{Rh}$, which are largely glutamatergic (Hur and Zaborsky, 2005). In light of its connections with both the hippocampus and mPFC (Vertes et al., 2006; Vertes et al., 2007), we hypothesized that Re/Rh critically mediate hippocampal-prefrontal synchrony during SWM. Of the putative pyramidal neurons that were included in entrainment analyses for rats that received muscimol in $\mathrm{Re} / \mathrm{Rh}$ (217/270 or $80 \%)$, a large proportion were significantly entrained to hippocampal theta during start-box occupancy ( $p<0.05$ for nonuniform spike-phase distribution; no-infusion baseline: $66.7 \%$, no-infusion testing: $65.9 \%$, saline baseline: $83.2 \%$, saline testing: $77.8 \%$, muscimol baseline: 91\%). In support of our hypothesis, Re/Rh inactivation dramatically decreased the proportion of mPFC neurons that phase-locked to hippocampal theta during delay periods (muscimol testing: 37.7\%; Fig. 10B). Due to differences in firing rate between baseline and testing epochs during muscimol sessions, bootstrapped Rayleigh's $z$-statistic and MRL values were derived from subsampled spike-phase distributions (see Materials and Methods). Distributions of subsampled Rayleigh's $z$-statistic values differed significantly between muscimol baseline and testing epochs (baseline $34.05 \pm 36.15$; testing $10.46 \pm 16.60$, means \pm SD; $D=0.51, p<0.001)$, but not between no-infusion $(D=0.23, p=0.18)$ or saline epochs $(D=0.27, p=0.08$, Kolmogorov-Smirnov tests). Subsampled MRL values were also significantly lower during muscimol testing than baseline epochs $(p=0.005)$, but there was no significant difference in MRL between no-infusion ( $p=0.1$ ) or saline ( $p=0.1$; Wilcoxon's ranksum tests) baseline and testing epochs (Fig. 10A).

Consistent with previous reports (Siapas et al., 2005; Sigurdsson et al., 2010), entrainment was strongest to past hip- 
A

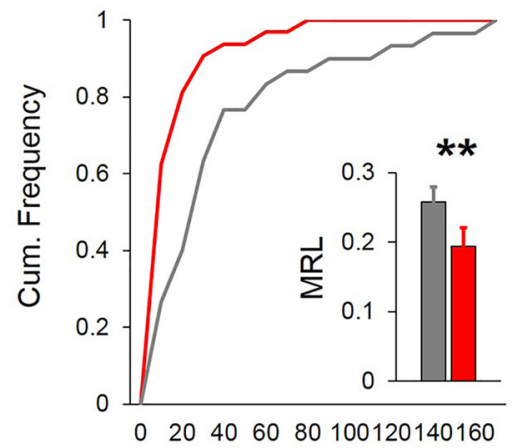

020406080100120140160

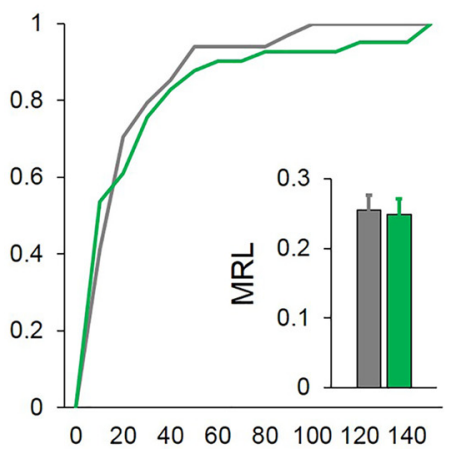

Rayleigh's Z-Statistic

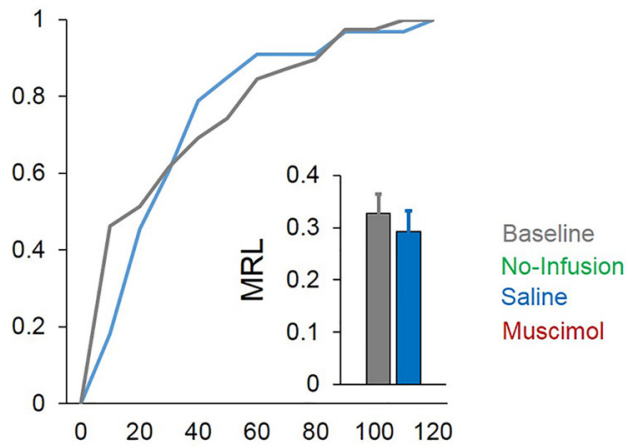

B

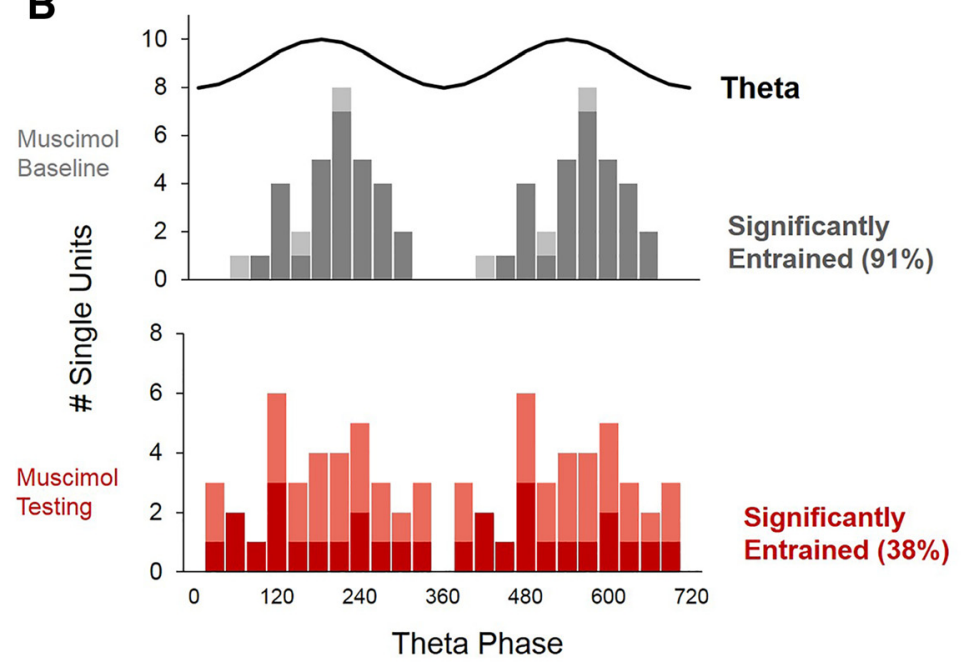

C

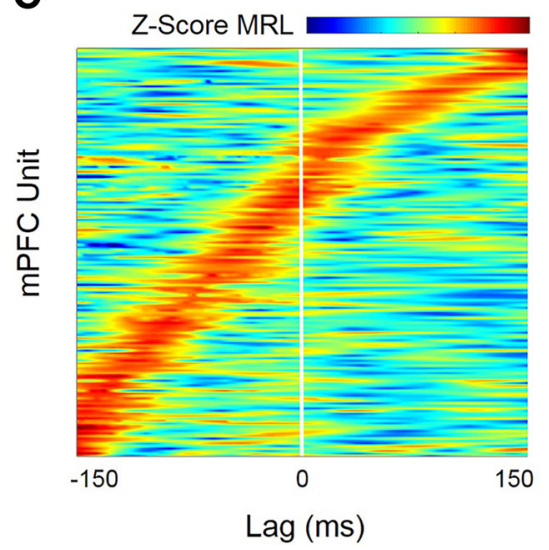

Figure 10. Muscimol infusions into Re/Rh decrease mPFC spike entrainment to the hippocampal theta oscillation during start-box occupancy. $\boldsymbol{A}$, Distributions of Rayleigh's Z-scores differ significantly between muscimol baseline and testing epochs (left), but no-infusion (middle) or saline baseline and testing epochs (right). Mean resultant vector length also differs significantly between muscimol baseline and testing epochs, but not no-infusion or saline baseline and testing epochs (insets). Inset bars indicate SEM. ${ }^{* *} p<0.01$ for Wilcoxon's rank-sum test. $\boldsymbol{B}$, Re/Rh inactivation significantly decreases the proportion of mPFC single units that show statistically significant phase locking to the hippocampal theta oscillation during start-box occupancy. Two theta cycles are plotted on the $x$-axis. $C$, The majority of single units in the mPFC maximally phase lock to past hippocampal theta phases (negative lag values) during start-box occupancy, indicating that dorsal hippocampal theta oscillations organize spike timing in the mPFC during the delay period of an SWM task.

pocampal theta phases during SWM-guided behavior. The majority of mPFC neurons (64.8\%), excluding those recorded during muscimol testing epochs, maximally phase locked to dorsal hippocampal theta phases of the past $(-37.67 \pm 93.26 \mathrm{~ms}$, mean temporal offset $\pm \mathrm{SD}, z=4.83, p<0.001$, signed-rank test with median 0 null; Fig. 10C). This effect was no longer observed for the population of mPFC neurons that significantly phase locked to hippocampal theta during start-box occupancy periods of muscimol testing epochs $(29.23 \pm 232.89 \mathrm{~ms}$, mean temporal offset $\pm \mathrm{SD}, z=0.714, p=0.48$, signed-rank test with medial 0 null; data not shown). These findings suggest that hippocampal theta oscillations organize spike timing in the MPFC selectively during SWM-guided behavior and that $\mathrm{Re} / \mathrm{Rh}$ are necessary for directionally specific interactions between the dorsal hippocampus and the mPFC during DA start-box occupancy.

Phase coherence

We next addressed the question of whether Re/Rh inactivation would interfere with hippocampal-mPFC synchrony by comparing phase coherence between baseline and testing epochs. We first found a main effect of frequency band between normalized (testing baseline) coherence scores only for the muscimol condition and selectively at the maze choice point (choice point:
$F_{(3,18)}=3.126, p=0.05$, start-box: $F_{(3,18)}=0.19, p=0.9$, stem: $F_{(3,18)}=1.223, p=0.33$, repeated-measures ANOVAs; Fig. 11A). Of the four frequency bands, delta coherence for theta was the only one significantly $<0\left(t_{(6)}=-4.107, p=0.006\right)$ during muscimol condition choice-point traversals $(p>0.1$ for other frequency bands, 1 -sample $t$ tests with 0 null). There were no significant differences in coherence across frequency bands for any other condition or maze location (no-infusion start box: $F_{(3,18)}=0.518, p=0.68$, stem: $F_{(3,18)}=0.034, p=0.99$, choice point: $F_{(3,18)}=0.163, p=0.92$, saline start box: $F_{(3,18)}=0.14, p=$ 0.94 , stem: $F_{(3,18)}=0.034, p=0.99$, choice point: $F_{(3,18)}=0.163$, $p=0.92$, repeated-measures ANOVAs). Finally, choice-pointnormalized theta coherence was significantly lower during muscimol conditions compared with other conditions $\left(F_{(2,12)}=\right.$ $14.348, p=0.001$, repeated-measures ANOVA; Fig. $11 C)$. This finding demonstrates that $\mathrm{Re} / \mathrm{Rh}$ inactivation caused frequencyspecific and location-specific decreases in choice-point thetaphase coherence that co-occurred with SWM impairments.

\section{Phase-amplitude coupling}

We next investigated whether $\mathrm{Re} / \mathrm{Rh}$ inactivation would decrease choice-point theta-gamma coupling between the hippocampus and mPFC. We found that normalized MI (testing ${ }_{\mathrm{MI}}-$ 
A

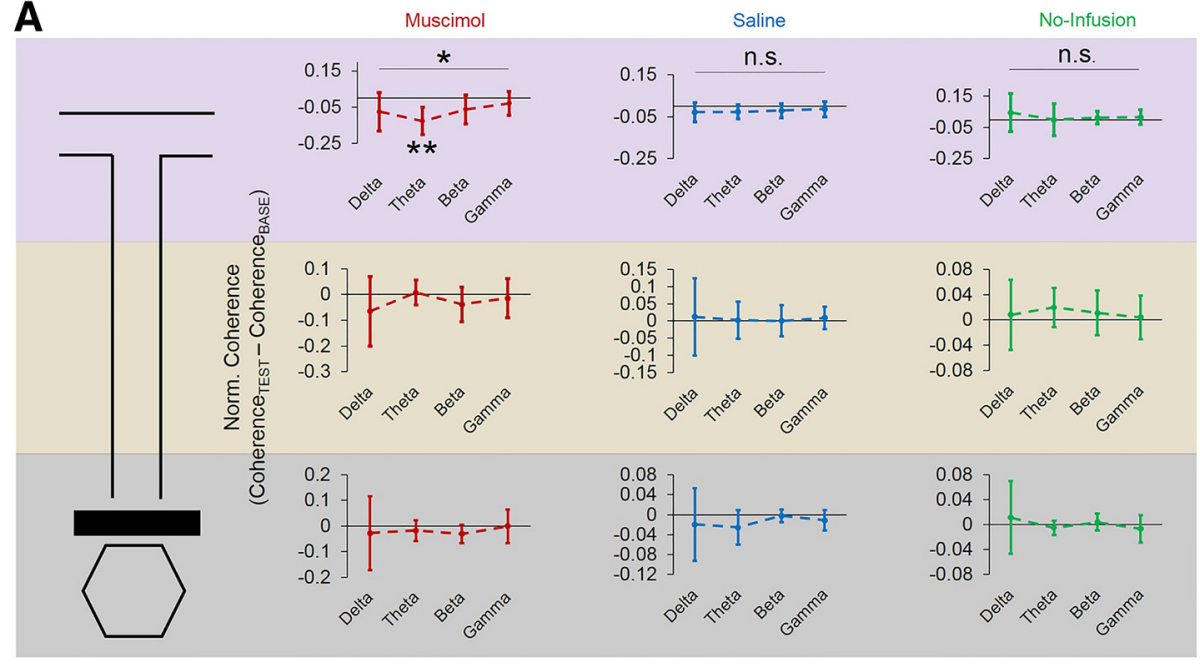

C

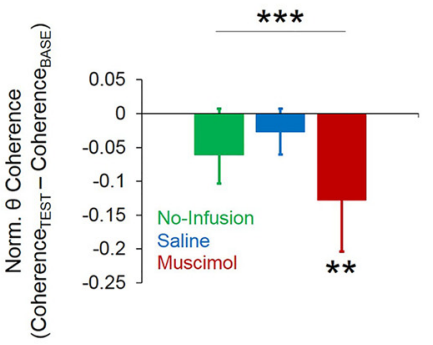

B

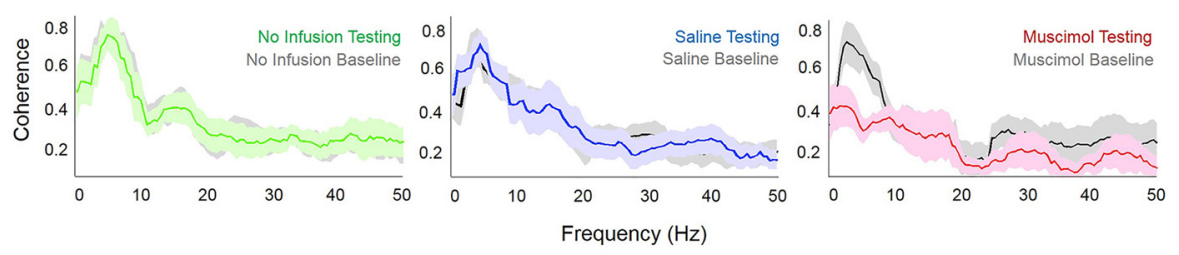

Figure 11. Re/Rh inactivation decreases hippocampal-prefrontal theta-phase coherence during choice-point traversals. $\boldsymbol{A}$, Normalized theta-phase coherence (testing - baseline) was significantly $<0$ only during muscimol sessions and only during choice-point traversals (top). Normalized phase coherence did not significantly differ from zero for any other frequency band or at any other maze location (middle panels, stem traversals; bottom panels, start-box occupancy). $\boldsymbol{B}$, Normalized theta-phase coherence at the choice point significantly differed between infusion conditions. C, Re/Rh inactivation caused theta-specific decreases in phase coherence during choice-point traversals (right), whereas no significant differences in theta-phase coherence were observed during no-infusion (left) and saline (middle) sessions. Reductions in theta coherence after Re/Rh inactivation peaked at $\sim 4 \mathrm{~Hz}$ and gradually declined until no significant differences were seen at $\sim 10 \mathrm{~Hz}$. Thick lines indicate means; shaded areas indicate SEM. For $\boldsymbol{A}$ and $\boldsymbol{B}$, error bars indicate means and $95 \%$ confidence intervals for 1 -sample $t$ tests with 0 null. ${ }^{*} p<0.05,{ }^{* *} p<0.01$, ${ }^{* * *} p<0.001$, n.s. $=$ no significant main effect of infusion condition for repeated-measures ANOVA.

baseline $_{\mathrm{MI}}$ ) during choice-point traversals was significantly lower during muscimol conditions $\left(F_{(2,12)}=8.847, p=0.004\right)$ compared with no-infusion $(p=0.05)$ and saline $(p=0.04)$ conditions; this effect did not exist for either stem $\left(F_{(2,12)}=1.208, p=\right.$ $0.33)$ or start-box $\left(F_{(2,12)}=0.182, p=0.84\right.$, repeated-measures ANOVAs) occupancy periods (Fig. 12A). Normalized MI during choice-point occupancy was significantly lower than zero only for muscimol conditions $\left(t_{(6)}=-3.73, p=0.01\right.$, 1-sample $t$ test; $p>0.24$ for other sessions). Re/Rh inactivation also decreased theta-gamma coupling within the MPFC during choice-point traversals. Choice-point normalized MI in the mPFC was significantly lower following muscimol infusions into $\operatorname{Re} / \mathrm{Rh}\left(F_{(2,12)}=\right.$ $17.672, p<0.001$, repeated-measures ANOVA) than during noinfusion $(p=0.03)$ and saline $(p=0.006)$ conditions and was also significantly $<0$ only for muscimol conditions $\left(t_{(6)}=\right.$ $-3.603, p=0.01,1$-sample $t$ test; $p>0.09$ for other conditions; Fig. 12A). As with theta-gamma coupling between the hippocampus and $\mathrm{mPFC}$, theta-gamma coupling within the mPFC was not affected by $\mathrm{Re} / \mathrm{Rh}$ inactivation during stem traversals $\left(F_{(2,12)}=\right.$ $0.365, p=0.7$, repeated-measures ANOVA) or start-box occupancy $\left(F_{(2,12)}=0.163, p=0.85\right.$, repeated-measures ANOVA). $\mathrm{Re} / \mathrm{Rh}$ inactivation did not affect hippocampal theta-gamma coupling between conditions at any maze location (choice point: $F_{(2,12)}=1.819, p=0.2$; stem: $F_{(2,12)}=0.509, p=0.51$; start box: $F_{(2,12)}=0.384, p=0.69$; all repeated-measures ANOVAs). These analyses show that phase-amplitude coupling at the choice point is dramatically disrupted by $\mathrm{Re} / \mathrm{Rh}$ inactivation, suggesting that $\mathrm{Re} / \mathrm{Rh}$ modulate phase synchrony between the dorsal hippocampus and mPFC during memory-guided decision making.

\section{Granger causality}

For Experiment 2, during choice-point traversals, only LI scores in the slow-gamma-frequency band were significantly $<0.5$ for all epochs (no-infusion baseline, $t_{(6)}=-12.174, p<0.001$; testing, $t_{(6)}=-15.052, p<0.001$; saline baseline, $t_{(6)}=-7.636, p<$ 0.001 ; testing, $t_{(6)}=-5.270, p=0.002$; muscimol baseline, $t_{(6)}=$ $-3.55, p=0.01,1$-sample $t$ tests with 0.5 null), excluding muscimol testing epochs $\left(t_{(6)}=0.126, p=0.9,1\right.$-sample $t$ test with 0.5 null; Fig. 13A). During start-box occupancy, LI scores solely in the theta-frequency band were significantly $>0.5$ for all epochs (no-infusion baseline, $t_{(6)}=5.138, p=0.004$; testing, $t_{(6)}=$ 7.649, $p<0.001$; saline baseline, $t_{(6)}=9.301, p<0.001$; testing, $t_{(6)}=4.417, p=0.004$; muscimol baseline, $t_{(6)}=6.244, p=$ $0.001,1$-sample $t$ tests with 0.5 null) with the exception of muscimol testing epochs $\left(t_{(6)}=-0.818, p=0.44,1\right.$-sample $t$ test with 0.5 null; Fig. $13 B$ ). Together with the granger causality data from Experiment 1, these data suggest that hippocampal to prefrontal communication in the theta-frequency band supports SWM during intertrial delay periods, prefrontal to hippocampal communication in the slow-gamma-frequency band supports SWM during choice-point traversals, and Re/Rh are necessary for bidirectional communication in the hippocampal-prefrontal circuit during SWM-guided decision making.

\section{Discussion}

We demonstrate here that SWM-specific behavior is encoded within ensembles of MPFC neurons and that SWM-specific population representations are accompanied by SWM-unique patterns of oscillatory synchrony between the dorsal hippocampus 


\section{B}
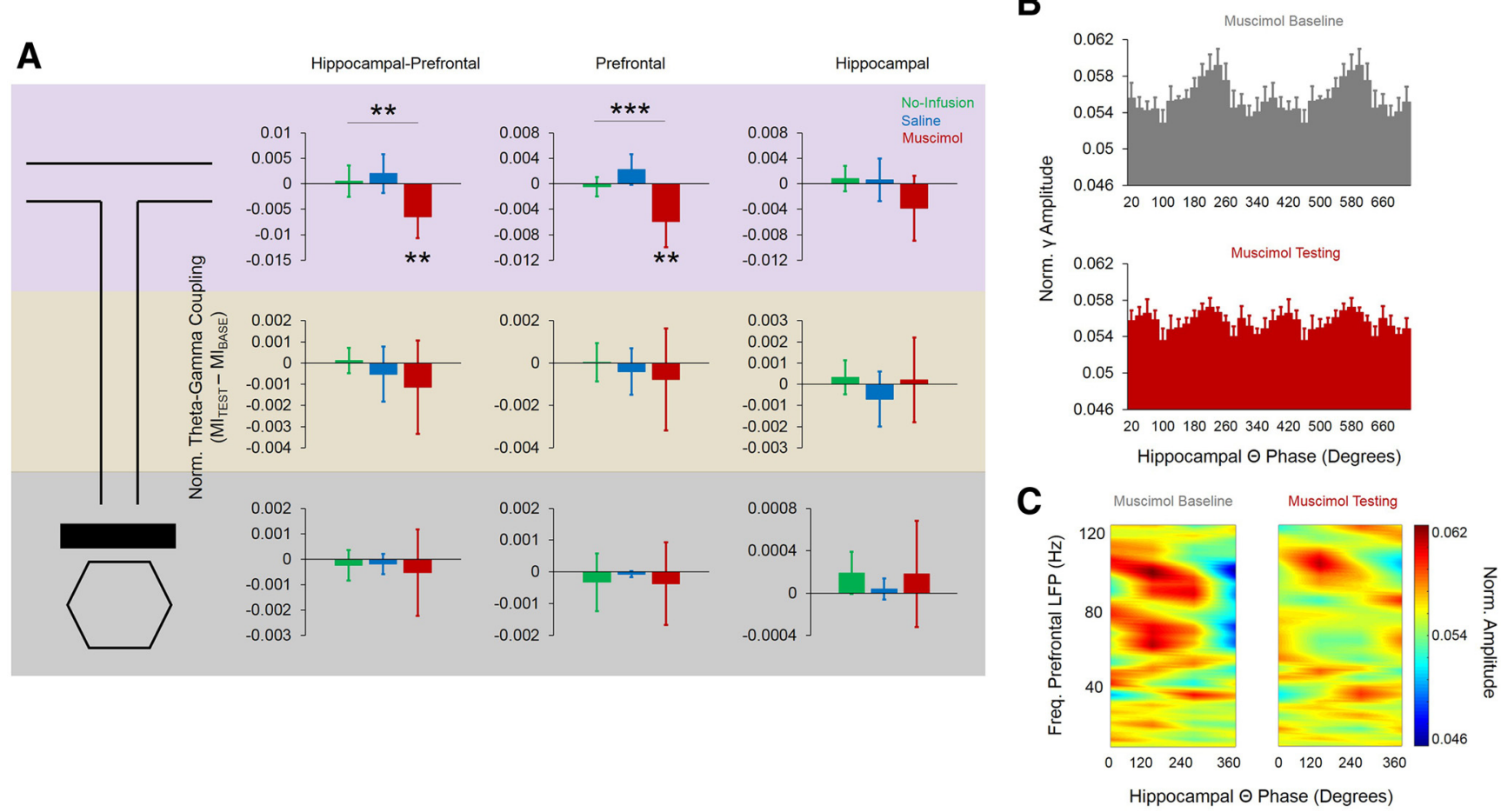

Figure 12. Re/Rh inactivation caused decreases in theta-gamma coupling during choice-point traversals. $\boldsymbol{A}$, Normalized theta-gamma coupling scores differed significantly between infusion conditions during choice-point traversals (top). This effect was specific to hippocampal-prefrontal (left) and prefrontal (middle), but not hippocampal (right), theta-gamma coupling. Error bars indicate means and $95 \%$ confidence intervals for 1 -sample $t$ tests with 0 null. ${ }^{* *} p<0.01,{ }^{* *} p<0.001$. B, Slow-gamma amplitude in the mPFC was modulated by hippocampal theta phase during muscimol baseline (top), but not testing (bottom), epochs. Error bars indicate SEM. Two theta cycles are plotted on the $x$-axis. C, Decreases in choice-point hippocampal-prefrontal theta-gamma coupling after Re/Rh inactivation are specific to the slow-gamma ( $\sim 50-80 \mathrm{~Hz})$-frequency band.

and $\mathrm{mPFC}$. We further show that the ventral midline thalamic nucleus reuniens $(\mathrm{Re})$ and rhomboid nucleus $(\mathrm{Rh})$, two nuclei that have reciprocal connections with the dorsal hippocampus and mPFC (Vertes et al., 2006; Vertes et al., 2007), are critical for both performance and hippocampal-prefrontal synchrony during SWM. These results implicate a triregional circuit in SWM and suggest that cortical subpopulations in the mPFC support SWM-guided decision making in part by virtue of their connectivity with a polysynaptic subcortical pathway between the dorsal hippocampus and ventral midline thalamus.

Many $\mathrm{mPFC}$ neurons showed firing rate patterns that distinctly represented the DA and CD tasks; importantly, these patterns were unique between neurons, indicating that SWMspecific behavior may not solely be represented by stereotyped variations in firing rate, but rather may be read out by patterns of summed prefrontal ensemble activity. In support of this notion, the task that an animal was performing could be predicted from decoded population activity in the MPFC at levels significantly above chance during both start-box occupancy and choice-point traversals. Importantly, task prediction was highly dependent on behavioral performance because decoding accuracy did not exceed chance levels when rats performed either task poorly. These results demonstrate that the MPFC dynamically encodes SWMspecific behavior with high-dimensional representations that are distributed among neuronal populations (Warden and Miller, 2010; Rigotti et al., 2013; Spellman et al., 2015).

How do mPFC ensembles represent mnemonic strategies on a behaviorally relevant timescale? One possibility is that prefrontal spiking is influenced by downstream inputs, which are themselves modulated by the behavioral context (in this case, the cognitive strategy that is necessary for successful task performance).
Many studies have linked the dorsal hippocampus with SWM in rodents (Dudchenko et al., 2000; Ainge et al., 2007; Czerniawski et al., 2009; Hallock et al., 2013a), making it a prime candidate for the provision of SWM-specific information to the mPFC. The results of the current study are consistent with research that has shown SWM-specific functional synchronization between the dorsal hippocampus and mPFC (Jones and Wilson, 2005; Hyman et al., 2010; Sigurdsson et al., 2010; O'Neill et al., 2013) and further implicates different patterns of hippocampal-prefrontal oscillatory synchrony during SWM-guided behavior. During intertrial delay periods, dorsal hippocampal theta oscillations organize $\mathrm{mPFC}$ spiking and predict changes in MPFC theta, suggesting that dorsal hippocampal theta may transmit information about a previous trial to the mPFC for the construction of a behaviorally relevant motor plan. During choice-point traversals, changes in slow-gamma oscillations in the mPFC predict changes in hippocampal slow gamma, suggesting that trial-specific information may be retrieved by the hippocampus from the mPFC when the animal makes an SWM-directed decision. Directionally specific interactions are accompanied by location and behaviorspecific phase synchrony during the DA task; specifically, singleunit entrainment during start-box occupancy and theta-phase coherence/theta-gamma phase-amplitude coupling at the choice point. The observation that neural activity in the hippocampalprefrontal circuit distinguishes between tasks at both the start box and choice point suggests that the SWM-guided decision making process is sequentially organized during each trial, with different components of this circuit exerting their contribution to decision making during distinct periods of each trial. Our results suggest that hippocampal-driven synchrony is most prominent 
A

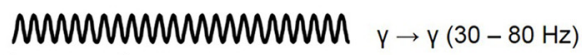

$\overline{100-267 \mathrm{~ms}}$
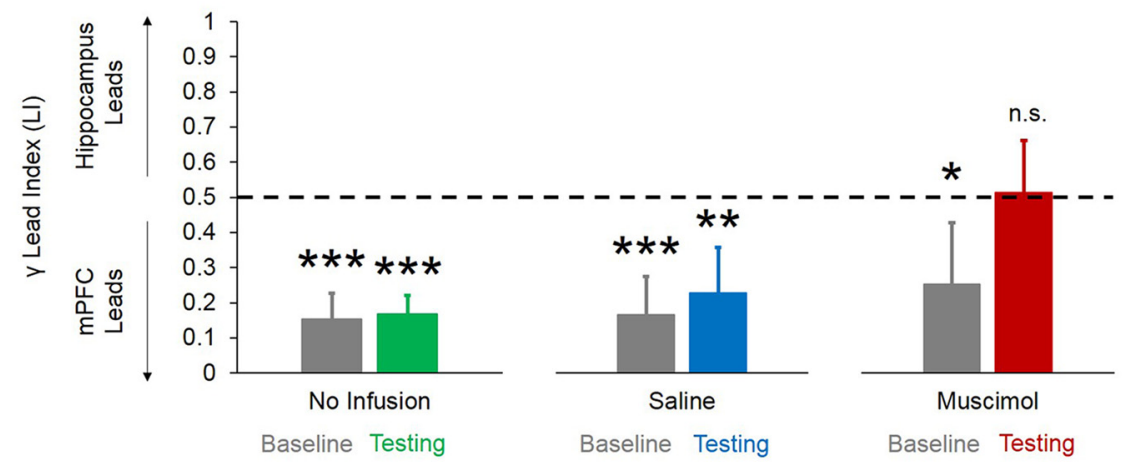

$\theta \rightarrow \theta(4-12 \mathrm{~Hz})$

B
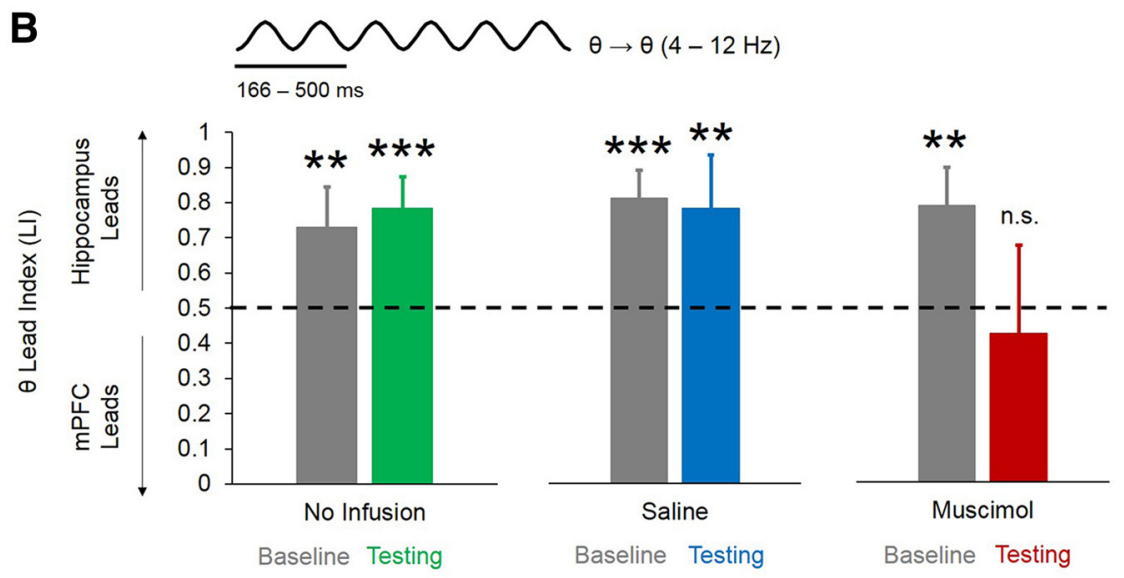
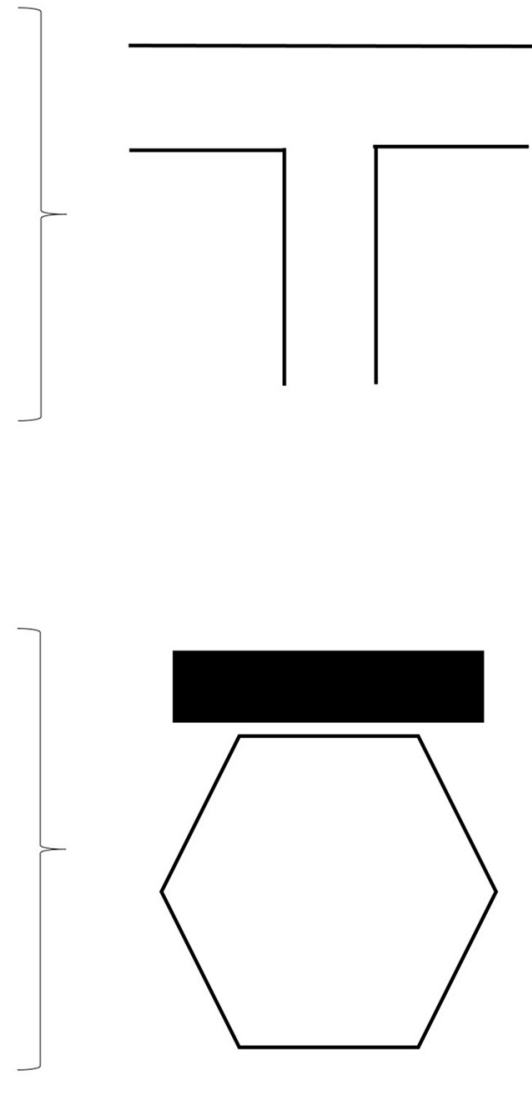

Figure 13. Re/Rh inactivation abolishes directionally specific interactions between the hippocampus and mPFC during DA performance. $\boldsymbol{A}$, mPFC leads the dorsal hippocampus in the slowgamma-frequency band during choice-point traversals. mPFC to hippocampal directionality during choice-point traversals is abolished after Re/Rh inactivation. $\boldsymbol{B}$, Dorsal hippocampus leads the $\mathrm{mPFC}$ in the theta-frequency band during start-box occupancy. Hippocampal to mPFC directionality during start-box occupancy is abolished after Re/Rh inactivation. Error bars indicate means and $95 \%$ confidence intervals for 1 -sample $t$ tests with 0.5 null. ${ }^{*} p<0.05,{ }^{* *} p<0.01,{ }^{* * *} p<0.001$, n.s. $=$ no significant effect of LI for 1 -sample $t$ test with 0.5 null.

earlier in the trial and, as the trial progresses, prefrontal-driven synchrony becomes more prominent.

Although prominent phase locking of mPFC single units to dorsal hippocampal theta oscillations was observed during SWM-specific intertrial delay periods, it is also a possibility that mPFC single-unit phase locking occurs at other points on the maze. In the current study, the limited amounts of spikes that occurred during stem and choice-point traversals precluded the analysis of single-unit entrainment at these maze locations. Therefore, we cannot exclude the possibility that mPFC single units also phase lock to hippocampal theta oscillations during other maze traversal periods. Mean phase-locking values were also higher during poor performance $\mathrm{CD}$ epochs relative to good performance DA epochs that were performed within the same dual-task session. These results suggest that rats may be using spatial working memory to locate the food reward during poor performance CD epochs, which would result in poor overall CD performance. In support of this interpretation, rats tended to alternate at significantly higher levels during poor CD performance and good DA performance compared with good CD performance and poor DA performance, further implicating high levels of hippocampal-prefrontal synchrony in SWM-guided behavior.

Previous studies have demonstrated that Re/Rh are necessary for SWM (Hembrook and Mair, 2011; Hembrook et al., 2012;
Hallock et al., 2013b), but did not demonstrate a relationship between Re/Rh function and hippocampal-prefrontal synchrony. Another study has shown that optogenetic silencing of $\mathrm{Re} / \mathrm{Rh}$ decreases trajectory coding in dorsal hippocampal neurons during continuous alternation task performance (Ito et al., 2015), suggesting that $\mathrm{Re} / \mathrm{Rh}$ relay route-specific information during goal-directed navigation. Critically, however, this study also did not measure the effect of Re/Rh silencing on interregional synchrony. We used an SWM-dependent DA task that requires both the dorsal hippocampus (Ainge et al., 2007; Hallock et al., 2013a) and Re/Rh to show that Re/Rh inactivation decreases dorsal hippocampal-prefrontal synchrony, which is hypothesized to be critical for SWM-guided behavior (Colgin, 2011; Gordon, 2011; Griffin, 2015). Given that behavior and synchrony changed simultaneously after Re/Rh inactivation, it is difficult to determine whether decreases in synchrony drove behavioral deficits or if behavioral deficits drove synchrony. In the current study, we opted to use a task that was dependent on Re/Rh so that we could make interpretations about the relationships among Re/Rh function, hippocampal-prefrontal synchrony, and SWM. One downfall to this approach, however, is that uncoupling hippocampal-prefrontal synchrony from behavior is difficult and precludes a conclusion about whether Re/Rh drives synchrony, behavior, or both. Further experiments will be necessary to de- 
termine causally directional relationships among SWM, hippocampal-prefrontal synchrony, and Re/Rh function.

In the current study, $\mathrm{Re} / \mathrm{Rh}$ inactivation produced deficits in SWM-guided behavior that co-occurred with decreases in SWMspecific patterns of oscillatory synchrony. Re/Rh inactivation decreased prefrontal spike entrainment to hippocampal theta oscillations during intertrial delays and decreased theta-gamma coupling and theta-phase coherence during choice-point traversals. Re/Rh inactivation also abolished directionally specific interactions between the hippocampus and mPFC. These results demonstrate that Re/Rh subserve SWM by modulating hippocampal-prefrontal synchrony and further show that Re/Rh are critical for bidirectional communication between the dorsal hippocampus and $\mathrm{mPFC}$ during SWM-guided behavior. One possible interpretation of these results is that $\mathrm{Re} / \mathrm{Rh}$ inactivation disrupts hippocampal-prefrontal communication while leaving independent hippocampal and prefrontal function intact. Another possibility is that $\mathrm{Re} / \mathrm{Rh}$ inactivation disrupts independent processing in the hippocampus, $\mathrm{mPFC}$, or both, which then indirectly disturbs hippocampal-prefrontal synchrony. These possibilities could be reconciled by using viral targeting strategies (e.g., DREADD receptors, optogenetics) to perturb Re/Rh afferent projections selectively in the hippocampus and mPFC to examine the independent contributions of $\mathrm{Re} / \mathrm{Rh}$ to either brain area. Future studies could use these techniques to gain a finer understanding of how $\mathrm{Re} / \mathrm{Rh}$ contributes to circuit function during SWM.

Projections from the dorsal hippocampus (via the dorsal subiculum) to Re/Rh (Varela et al., 2014) may support information maintenance during intertrial delay periods and afferents from the mPFC to the ventral midline thalamus may support the retrieval of trial-specific information during the execution of an SWM-directed motor outcome. Although the nucleus reuniens receives projections from the dorsal subiculum, injections of cholera toxin B (a retrograde tracer) into Re/Rh showed that the density of reuniens projections increases at more posterior levels of the subiculum (Varela et al., 2014). This raises the possibility that reductions in hippocampal-prefrontal synchrony seen after $\mathrm{Re} / \mathrm{Rh}$ inactivation in the current study were at least partially due to a disruption of ventral subicular inputs to $\mathrm{Re} / \mathrm{Rh}$, which would point to a role for $\mathrm{Re} / \mathrm{Rh}$ in mediating ventral and dorsal hippocampal-prefrontal synchrony. In support of this notion, a previous study has demonstrated that muscimol infusions into the ventral hippocampus disrupt dorsal hippocampal-prefrontal synchrony during SWM (O'Neill et al., 2013). In addition to the hippocampal-thalamo-prefrontal pathway that was investigated in the current study, previous studies have also demonstrated that direct monosynaptic projections from the ventral hippocampus to the mPFC support directly the encoding, but not maintenance or retrieval, of spatial information during the sample phase of a DNMP T-maze task (Spellman et al., 2015), suggesting that distinct pathways between the hippocampus and mPFC transmit SWM-specific information at different stages of the SWM-guided decision-making process. Together, these results highlight the complexity of the neural circuitry that regulates SWM. Nevertheless, the results of the current study contribute to our understanding of the neural mechanisms that support SWM by confirming that the ventral midline thalamus critically contributes to SWM-directed task performance and dorsal hippocampal-prefrontal synchrony, providing novel insight into how the hippocampal-thalamo-prefrontal circuit functions at the intersection of cognition and action.

\section{References}

Ainge JA, van der Meer MA, Langston RF, Wood ER (2007) Exploring the role of context-dependent hippocampal activity in spatial alternation behavior. Hippocampus 17:988-1002. CrossRef Medline

Allen TA, Narayanan NS, Kholodar-Smith DB, Zhao Y, Laubach M, Brown $\mathrm{TH}$ (2008) Imaging the spread of reversible brain inactivations using fluorescent muscimol. J Neurosci Methods 171:30-38. CrossRef Medline

Brandon MP, Bogaard AR, Libby CP, Connerney MA, Gupta K, Hasselmo ME (2011) Reduction of theta rhythm dissociates grid cell spatial periodicity from directional tuning. Science 332:595-599. CrossRef Medline

Buzsaki G (2006) Rhythms of the brain. New York: OUP.

Churchwell JC, Kesner RP (2011) Hippocampal-prefrontal dynamics in spatial working memory: interactions and independent parallel processing. Behav Brain Res 225:389-395. CrossRef Medline

Colgin LL (2011) Oscillations and hippocampal-prefrontal synchrony. Curr Opin Neurobiol 21:467-474. CrossRef Medline

Czerniawski J, Yoon T, Otto T (2009) Dissociating space and trace in dorsal and ventral hippocampus. Hippocampus 19:20-32. CrossRef Medline

Dudchenko PA, Wood ER, Eichenbaum H (2000) Neurotoxic hippocampal lesions have no effect on odor span and little effect on odor recognition memory but produce significant impairments on spatial span, recognition, and alternation. J Neurosci 20:2964-2977. Medline

Fell J, Axmacher N (2011) The role of phase synchronization in memory processes. Nat Rev Neurosci 12:105-118. CrossRef Medline

Fellows BJ (1967) Chance stimulus sequences for discrimination tasks. Psych Bull 67:87-92. CrossRef

Ferino F, Thierry AM, Glowinski J (1987) Anatomical and electrophysiological evidence for a direct projection from Ammon's horn to the medial prefrontal cortex in the rat. Exp Brain Res 65:421-426. Medline

Gordon JA (2011) Oscillations and hippocampal-prefrontal synchrony. Curr Opin Neurobiol 21:486-491. CrossRef Medline

Griffin AL (2015) Role of the thalamic nucleus reuniens in mediating interactions between the hippocampus and medial prefrontal cortex during spatial working memory. Front Syst Neurosci 9:29. CrossRef Medline

Griffin AL, Hallock HL (2013) Hippocampal signatures of episodic memory: evidence from single-unit recording studies. Front Behav Neurosci 7:54. CrossRef Medline

Hallock HL, Griffin AL (2013) Dynamic coding of dorsal hippocampal neurons between tasks that differ in structure and memory demand. Hippocampus 23:169-186. CrossRef Medline

Hallock HL, Arreola AC, Shaw CL, Griffin AL (2013a) Dissociable roles of the dorsal striatum and dorsal hippocampus in conditional discrimination and spatial alternation T-maze tasks. Neurobiol Learn Mem 100: 108-116. CrossRef Medline

Hallock HL, Wang A, Shaw CL, Griffin AL (2013b) Transient inactivation of the thalamic nucleus reuniens and rhomboid nucleus produces deficits of a working-memory dependent tactile-visual conditional discrimination task. Behav Neurosci 127:860-866. CrossRef Medline

Hembrook JR, Mair RG (2011) Lesions of reuniens and rhomboid thalamic nuclei impair radial maze win-shift performance. Hippocampus 21: 815-826. CrossRef Medline

Hembrook JR, Onos KD, Mair RG (2012) Inactivation of ventral midline thalamus produces selective spatial delayed conditional discrimination impairment in the rat. Hippocampus 22:853-860. CrossRef Medline

Herkenham M (1979) The afferent and efferent connections of the ventromedial thalamic nucleus in the rat. J Comp Neurol 183:487-517. CrossRef Medline

Hur EE, Zaborszky L (2005) Vglut2 afferents to the medial prefrontal and primary somatosensory cortices: a combined retrograde tracing in situ hybridization study. J Comp Neurol 483:351-373. CrossRef Medline

Hyman JM, Zilli EA, Paley AM, Hasselmo ME (2010) Working memory performance correlates with prefrontal-hippocampal theta interactions but not with prefrontal neuron firing rates. Front Integr Neurosci 4:2. CrossRef Medline

Ito HT, Zhang SJ, Witter MP, Moser EI, Moser MB (2015) A prefrontalthalamo-hippocampal circuit for goal-directed spatial navigation. Nature 522:50-55. CrossRef Medline

Jarvis MR, Mitra PP (2001) Sampling properties of the spectrum and coherency of sequences of action potentials. Neural Comput 13:717-749. CrossRef Medline

Jay TM, Glowinski J, Thierry AM (1989) Selectivity of the hippocampal 
projection to the prelimbic area of the prefrontal cortex in the rat. Brain Res 505:337-340. CrossRef Medline

Jones MW, Wilson MA (2005) Theta rhythms coordinate hippocampalprefrontal interactions in a spatial memory task. PLoS Biol 3:e402. CrossRef Medline

Kopp F, Schröger E, Lipka S (2006) Synchronized brain activity during rehearsal and short-term memory disruption by irrelevant speech is affected by recall mode. Int J Psychophysiol 61:188-203. CrossRef Medline

Lawrie SM, Buechel C, Whalley HC, Frith CD, Friston KJ, Johnstone EC (2002) Reduced frontotemporal functional connectivity in schizophrenia associated with auditory hallucinations. Biol Psychiatry 51:10081011. CrossRef Medline

Lee I, Kesner RP (2003) Time-dependent relationship between the dorsal hippocampus and the prefrontal cortex in spatial memory. J Neurosci 23:1517-1523. Medline

Meyer-Lindenberg AS, Olsen RK, Kohn PD, Brown T, Egan MF, Weinberger DR, Berman KF (2005) Regionally specific disturbance of dorsolateral prefrontal-hippocampal functional connectivity in schizophrenia. Arch Gen Psychiatry 62:379-386. CrossRef Medline

O'Neill PK, Gordon JA, Sigurdsson T (2013) Theta oscillations in the medial prefrontal cortex are modulated by spatial working memory and synchronize with the hippocampus through its ventral subregion. J Neurosci 33:14211-14224. CrossRef Medline

Paxinos J, Watson C (2005) The rat brain in stereotaxic coordinates, Ed 5. New York: Elsevier.

Payne L, Kounios J (2009) Coherent oscillatory networks supporting shortterm memory retention. Brain Res 1247:126-132. CrossRef Medline

Rigotti M, Barak O, Warden MR, Wang XJ, Daw ND, Miller EK, Fusi S (2013) The importance of mixed selectivity in complex cognitive tasks. Nature 497:585-590. CrossRef Medline

Sarnthein J, Petsche H, Rappelsberger P, Shaw GL, von Stein A (1998) Synchronization between prefrontal and posterior association cortex during human working memory. Proc Natl Acad Sci U S A 95:7092-7096. CrossRef Medline

Schmitzer-Torbert N, Jackson J, Henze D, Harris K, Redish AD (2005)
Quantitative measures of cluster quality for use in extracellular recordings. Neuroscience 131:1-11. CrossRef Medline

Serrien DJ, Pogosyan AH, Brown P (2004) Influence of working memory on patterns of motor related cortico-cortical coupling. Exp Brain Res 155: 204-210. CrossRef Medline

Siapas AG, Lubenov EV, Wilson MA (2005) Prefrontal phase locking to hippocampal theta oscillations. Neuron 46:141-151. CrossRef Medline

Sigurdsson T, Stark KL, Karayiorgou M, Gogos JA, Gordon JA (2010) Impaired hippocampal-prefrontal synchrony in a genetic mouse model of schizophrenia. Nature 464:763-767. CrossRef Medline

Spellman T, Rigotti M, Ahmari SE, Fusi S, Gogos JA, Gordon JA (2015) Hippocampal-prefrontal input supports spatial encoding in working memory. Nature 522:309-314. CrossRef Medline

Swanson LW (1981) A direct projection from Ammon's horn to prefrontal cortex in the rat. Brain Res 217:150-154. CrossRef Medline

Thierry AM, Gioanni Y, Dégénétais E, Glowinski J (2000) Hippocampoprefrontal cortex pathway: Anatomical and electrophysiological characteristics. Hippocampus 10:411-419. CrossRef Medline

Tort AB, Komorowski R, Eichenbaum H, Kopell N (2010) Measuring phase-amplitude coupling between neuronal oscillations of different frequencies. J Neurophysiol 104:1195-1210. CrossRef Medline

Varela C, Kumar S, Yang JY, Wilson MA (2014) Anatomical substrates for direct interactions between hippocampus, medial prefrontal cortex, and the thalamic nucleus reuniens. Brain Struct Funct 219:911-929. CrossRef Medline

Vertes RP, Hoover WB, Do Valle AC, Sherman A, Rodriguez JJ (2006) Efferent projections of reuniens and rhomboid nuclei of the thalamus in the rat. J Comp Neurol 499:768-796. CrossRef Medline

Vertes RP, Hoover WB, Szigeti-Buck K, Leranth C (2007) Nucleus reuniens of the midline thalamus: Link between the medial prefrontal cortex and the hippocampus. Brain Res Bull 71:601-609. CrossRef Medline

Warden MR, Miller EK (2010) Task-dependent changes in short-term memory in the prefrontal cortex. J Neurosci 30:15801-15810. CrossRef Medline 